University of Louisville

ThinkIR: The University of Louisville's Institutional Repository

Electronic Theses and Dissertations

$12-2005$

\title{
Thermal conductivity measurement of thin film of carbon SWNT using the $3 w$ technique.
}

Anton Nickolaevich Sidorov 1981-

University of Louisville

Follow this and additional works at: https://ir.library.louisville.edu/etd

\section{Recommended Citation}

Sidorov, Anton Nickolaevich 1981-, "Thermal conductivity measurement of thin film of carbon SWNT using the 3w technique." (2005). Electronic Theses and Dissertations. Paper 1326.

https://doi.org/10.18297/etd/1326

This Master's Thesis is brought to you for free and open access by ThinkIR: The University of Louisville's Institutional Repository. It has been accepted for inclusion in Electronic Theses and Dissertations by an authorized administrator of ThinkIR: The University of Louisville's Institutional Repository. This title appears here courtesy of the author, who has retained all other copyrights. For more information, please contact thinkir@louisville.edu. 


\title{
THERMAL CONDUCTIVITY MEASUREMENT OF THIN

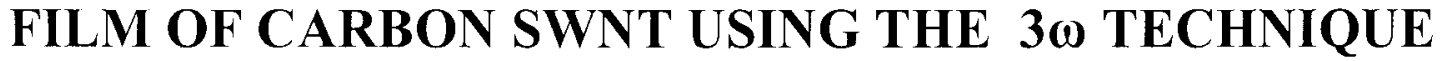

\author{
By \\ Anton Nickolaevich Sidorov \\ M.S., Perm State University, Russia 2003
}

\begin{abstract}
A Thesis
Submitted to the Faculty of the

Graduate School of the University of Louisville

in Partial Fulfillment of the Requirements

for the Degree of
\end{abstract}

Master of Science

Department of Physics

University of Louisville

Louisville, Kentucky

December 2005 


\title{
THERMAL CONDUCTIVITY MEASUREMENT OF THIN FILM OF CARBON SWNT USING THE $3 \omega$ TECHNIQUE
}

\author{
By \\ Anton Nickolaevich Sidorov \\ M.S., Perm Sate University, Russia 2003
}

A Thesis approved on

November 2005

By the Following Reading Committee:

Dr. Gamini Sumanasekera, Thesis Director

Dr. Shi Yu Wu

Dr. Kevin Walsh 


\section{ACKNOWLEDGEMENTS}

I would like to thank my major professor, Dr. Gamini Sumanasekera, for his guidance and patience. I would also like to thank the other committee members, Dr. Shi Yu Wu and Dr Kevin Walsh, for their classes and assistance over the past two years. I would also like to express my thanks to my parents for their understanding and patience during that time when I was doing my master degree. Also I acknowledge with pleasure and gratitude to all the people in the Physics department, ECE department, and the Physics nano-lab who assisted me and gave me moral support while editing this thesis. 


\title{
ABSTRACT \\ THERMAL CONDUCTIVITY MEASUREMENT OF THIN FILM OF CARBON SWNT USING THE 3W TECHNIQUE
}

\author{
Anton Nickolaevich Sidorov
}

November 2005

We have measured the temperature-dependent thermal conductivity, $\kappa(T)$ of single walled carbon nanotubes from $300 \mathrm{~K}$ to $8 \mathrm{~K}$ by $3 \omega$ method. $\kappa(T)$ exhibits linear temperature dependence in the entire temperature range. Our results are in good agreement with the results obtained by Hone et al. using a standard steady state technique. Also we measured the temperature-dependent thermal conductivity $\kappa(T)$ of quartz and $\mathrm{SiO}_{2}$ in the same temperature range ( $300 \mathrm{~K}$ to $8 \mathrm{~K}$ ) as a standardization tool. Our technique shows promise for the thermal conductivity measurements of many nanostructures with slight modifications. 


\section{TABLE OF CONTENTS}

Page

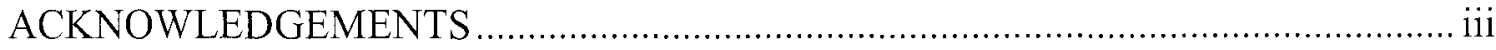

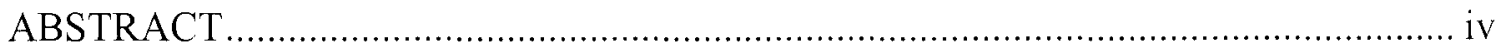

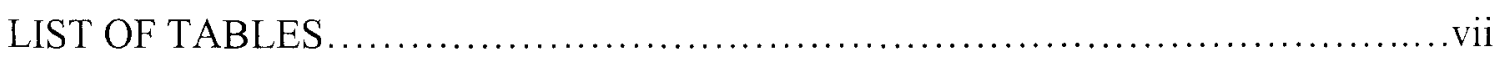

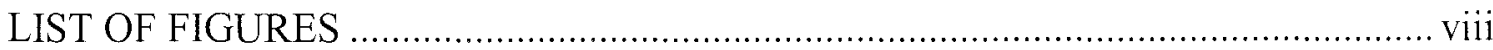

CHAPTER

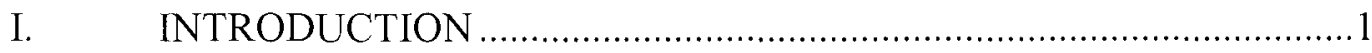

1. Nanoscience and Nanotechnology ................................................

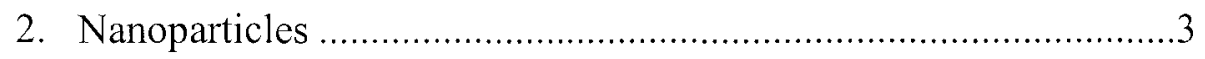

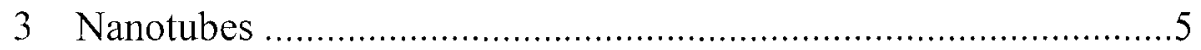

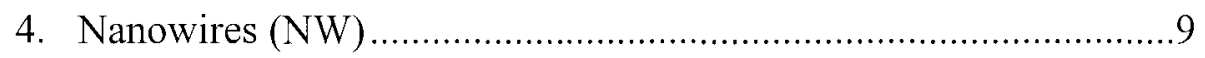

II. THEORETICAL BASIS OF $3 \omega$ METHOD

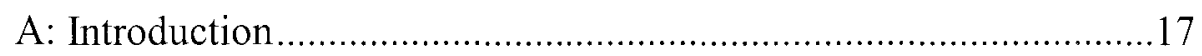

B: Theoretical basis of $3 \omega$ method ............................ 20

1: The $1 \mathrm{D}$ heat conduction equation and its solution..........................21

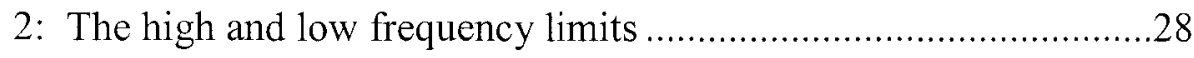

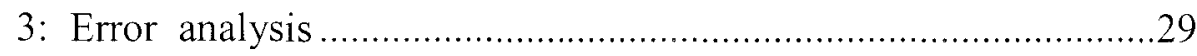

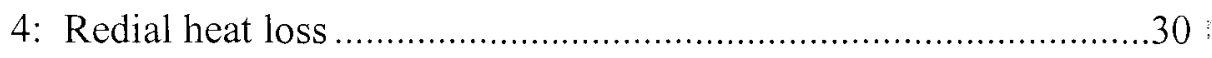

III. EXPERIMENT 


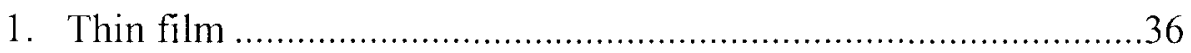

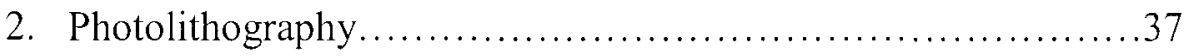

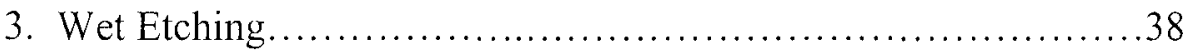

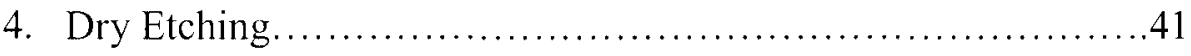

5. Lift Off.................................................

6. Mask design..........................................43

7. Software for maskdesign...............................43

8. Alignment marks .........................................44

9. Experiment.........................................48

10. Results and Discussion.................................53

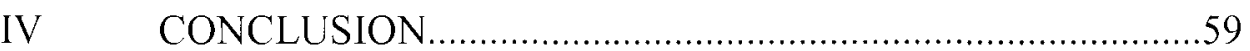

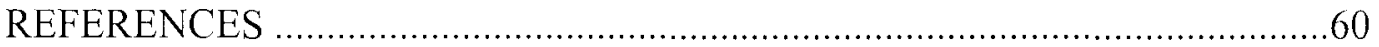

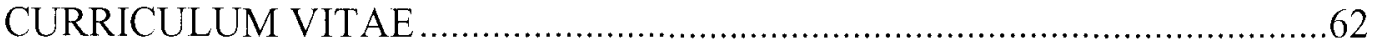




\section{LIST OF TABLES}

TABLE

PAGE

1. The growth parameters of some nanowires.................................. 12

2. $\kappa(\mathrm{T})$ of SWNT thin film and quartz sample...................................... 


\section{LIST OF FIGURES}

FIGURE

1. Gold Nanoparticles by laser ablation in aqueous solution of surfactant.......................4

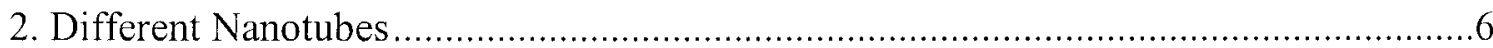

3. Single wall carbon field effect transistor ......................................................

4. AFM image of CNT in a carbon-nanotube ...................................................... 8

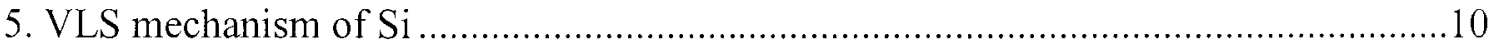

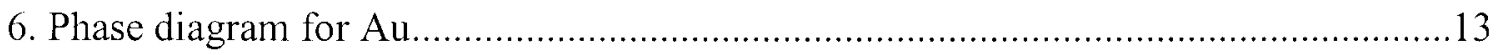

7. TEM and FETEM image of GaAs NW ...................................................... 14

8. TEM image of GaAs NW ............................................................................. 15

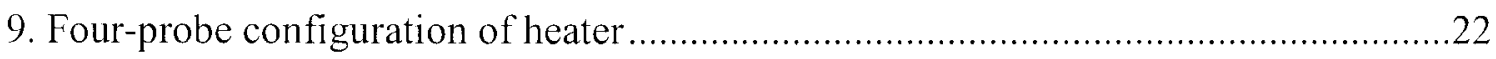

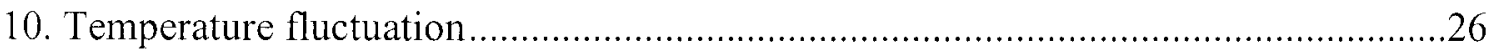

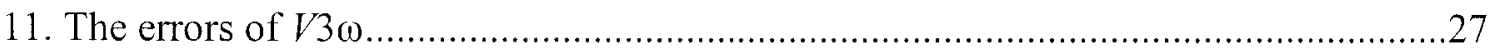

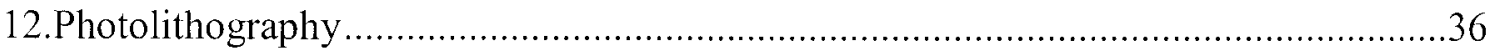

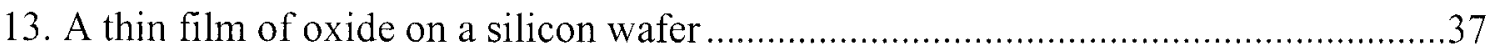

14. The simplest structures that can be formed using $\mathrm{KOH}$ to etch a silicon wafer with the

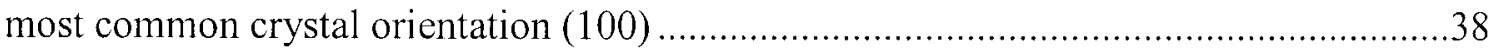

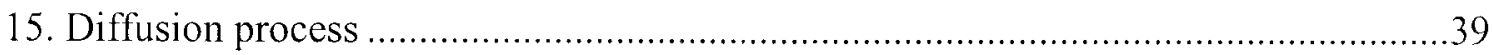

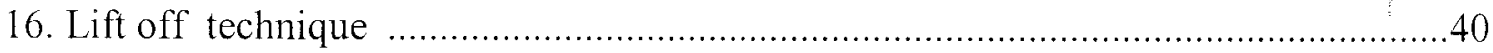

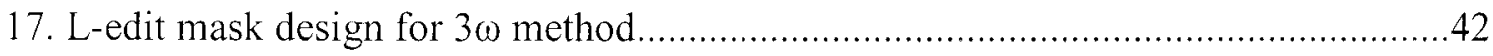




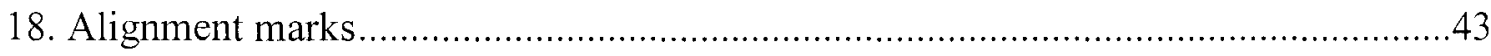

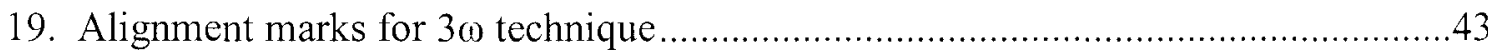

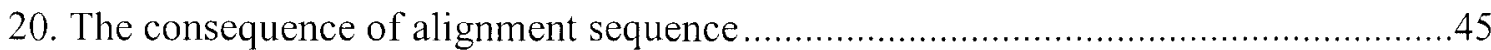

21.Block diagram of $3 \omega$ experiment ..................................................................

22. Geometry of the 4 pad test structure for the $3 \omega$ method ......................................49

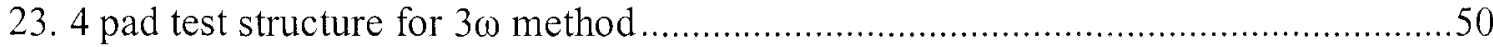

24. Schematic diagram of the measurement system ...................................................50

25. Thermal conductivity coefficient $\lambda$ of quartz versus temperature $T$........................52

26. For $\mathrm{Si}$ and $\mathrm{SiO} 2$. Temperature versus Frequency ...........................................52

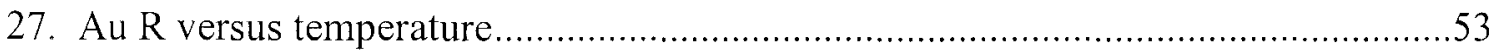

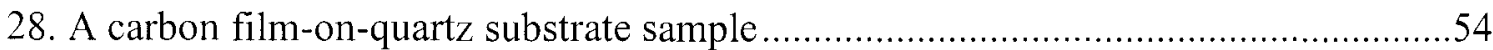

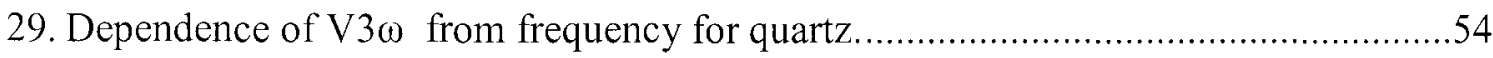

30. V3 $\omega$ versus $\log ($ freq) for Carbon thin film at room temperature..........................55

31. $\lambda$ carbon thin film versus temperature.......................................... 56

32. Temperature-dependent thermal conductivity of single-walled nanotubes by thermocouple method from J.Hone experiment..............................57

33.A front panel of LabView 6.2 program which has been creating for $3 \omega$

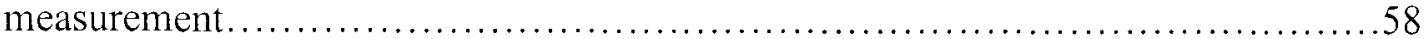




\section{CHAPTER I}

\section{INTRODUCTION}

\section{Nanoscience and Nanotechnology}

Nanophase and nanostructured materials have attracted a great deal of interest due to their potential applications in different areas such as electronics, optics, catalysis, ceramics, magnetic data storage, and nanocomposites. The unique properties of nanomaterials are determined by their size, surface structures, interparticle interaction and surface to volume ratio.

Nanomaterials are classified into 1) Nanostructured materials, such as nanowires, nanotubes, nanowebs and thin films, and 2) Nanophase materials like nanoparticles and catalysts. To distinguish nanomaterials from bulk it is important to study the unique properties of nanomaterials and their applications in science and technology.

A parameter introduced by nanomaterials is the surface/interface to volume ratio. A high percentage of surface atoms lead to many size dependent phenomena. The finite size of the particle confines the spatial distribution of electrons and causes quantized energy levels due to small size effect. This can provide deep insight into the theory of energy transitions from discrete energy levels for fundamental elements and continuum energy levels for bulk materials. This quantum confinement has applications in semiconductors, optoelectronics and non-linear optics. Another fundamental feature of nanoscience is nucleation. Nucleation and growth are two important parameters for synthesis of thin 
films and nanowires resulting in formation of crystalline particles under suitable conditions. Aggregation of these particles leads to nanocrystalline structures.

Nanomaterials have novel mechanical properties. The strength of a solid depends on its size and surface to volume ratio. In a crystalline structure strength is dependent on grain boundary sliding and energy dissipation at interfaces. By decreasing the size of the grain the hardness of the material is increased. The high surface to volume ratio determines the thermodynamic properties of the materials. A structure with less coordinated surface atoms increases the surface energy so that the diffusion of atoms happens at lower temperatures. As a result the melting temperature decreases in nanomaterials. For example, the melting temperature for bulk gold is $1063{ }^{\circ} \mathrm{C}$, but for gold nanoparticles is about $30{ }^{\circ} \mathrm{C}$. Semiconductor nano dots are zero dimensional quantum systems, in which carriers are confined and convert the density of states to a set of discrete quantum levels which is necessary for semiconductor laser function. In nanodevices quantum physics is substituted for classical physics and devices work based on tunneling of the electrons through potential barriers and a single electron can govern the function of a transistor, switch or memory element. The discovery of $\mathrm{C}_{60}$ (Fullerene) is another new field in carbon related nanomaterials. Carbon nanotubes are concentric cylinders of graphite sheets and they appear as single cylinders called single wall carbon nanotubes (SWNT), as two concentric cylinders called double wall carbon nanotubes (DWNT), or multi concentric tubes called multi wall carbon nanotubes (MWNT). The finite dimension of carbon nanotubes and the chirality angle, which determines how the graphite sheet is rolled, result in unique electronic properties; such as ballistic quantum conductance, semiconductor junctions, and electron field emission. The structure of carbon nanotubes 
leads to an extraordinary mechanical strength and high elastic strength. There are numerous applications for nanotubes related to their structure and properties. They can be added to a chain of polymer in different ways or used a fine tip for Atomic force microscopy (AFM) or scanning tunneling microscopy (STM). There are a vast number of projects in progress to apply carbon nanotubes for gas sensor or electronic devices and transistors. [1]

There are three important steps in nanotechnology; material preparation, property measurement and device fabrication. Nanomaterials such as nano particles, nanowebs, nanorods, nanowires, nanotubes and more nanostructures have been prepared and some of the nanostructures have been characterized.

\section{Nanoparticles}

Nanoparticles are currently made of a wide variety of materials. One of the new generations of materials is ceramics, which are metal oxides such as titanium, zinc, aluminum and iron oxides, as well as metal nanoparticles. Silicate nanoparticles, a kind of ceramic nanoparticle, are flakes about $1 \mathrm{~nm}$ thick and 100 to $1000 \mathrm{~nm}$ across. The nanoparticles can be incorporated into polymers during polymerization or by melt compounding. Pure metal nanoparticles can be merged into a solid by chemical reaction of a solvent and substrate; these nanoparticles create a thin layer or a film.

Mass production of nanoparticles is important in nanoelectronics, for example, insulating matrices encapsulating metallic nanoparticles exhibit nonlinear optical properties which are size dependent. Any synthesis technique for particles to be used in these applications 
must produce high quality, individual, nanoparticles with narrow size distributions (mono- dispersed). Spherical, high quality, nanometer sized particles can be made from a wide variety of materials via laser ablation of micro particles (LAM). The finite volume contained within a target microparticle significantly increases the importance of the breakdown-induced shock wave in nanoparticle formation [2]. The nanoparticles can be obtained via other methods of laser ablation for example in an aqueous solution [3].(Fig.1) There are other techniques for synthesizing nanoparticles. For example; condensation from a vapor, chemical synthesis and solid-state processes such as mechanical milling. Particles can be coated then with hydrophobic or hydrophilic material, depending on the application.

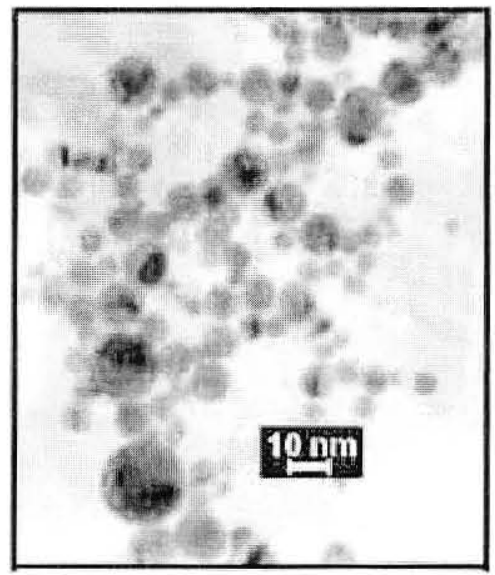

Fig.1 Gold Nanoparticles by laser ablation in aqueous solution of surfactant

The vapor condensation method involves evaporation of a solid metal followed by rapid condensation to form nanosize clusters constituting powder. To obtain metal particles only inert gas is applied, whereas a reactive oxygen atmosphere is applied for metal oxide particles. Another method of the vapor condensation technique is vacuum evaporation on running liquid. This technique utilizes a thin film of viscous material, oil, or a polymer on 
a rotating plate in a vacuum gauge; the material evaporates or sputters nanoparticles into a vacuum. Another possible method for vapor condensation nanoparticle synthesis is the chemical vapor deposition technique. Both liquid and gas are placed in the reactor and the reactor is heated for a specific time depending on the desired shape of the particles. The chemical synthesis technique essentially consists of growing nanoparticles in a liquid medium composed of various reactants. This method is also used to create quantum dots. An example of the solid-state method is milling or grinding to create nanoparticles. The milling time and medium affect the resultant nanoparticles [4].

\section{Nanotubes}

Carbon nanotubes are unique nanostructures with remarkable electronic and mechanical properties. Nanotubes can behave like one-dimensional quantum wires. The structure of a nanotube is a hexagonal network of carbon atoms that has been rolled up to make a seamless cylinder. The cylinder typically has a diameter of approximately one nanometer and length of tens of microns. Each end is "capped" with half of a fullerene molecule. Single-wall nanotubes can be thought of as the fundamental cylindrical structure, and form the building blocks of both multi-wall nanotubes and ordered bundles of single-wall carbon nanotubes called ropes. Many theoretical studies have predicted the properties of single-wall nanotubes. As shown (Fig.2), three types of nanotubes are possible, called "armchair", "zigzag" and "chiral" nanotubes, depending on how the two-dimensional graphene sheet is "rolled up". 


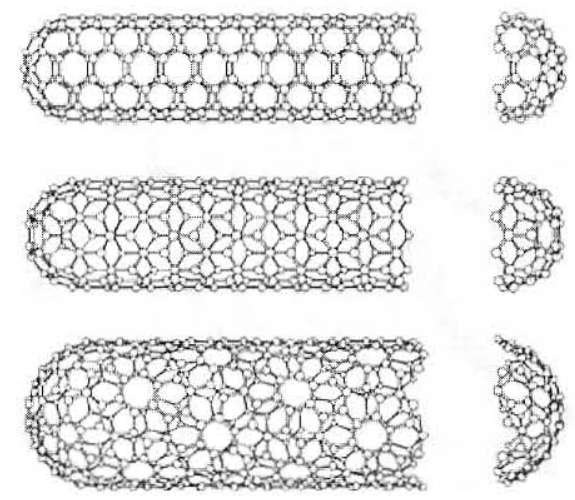

Fig.2 It is shown here a $(5,5)$ armchair nanotube (Top), a $(9,0)$ zigzag nanotube (middle) and a $(10,5)$ chiral nanotube. The diameter of the nanotubes depends on the values of $n$ and $m$.

The diameter and the chiral angle of the nanotube have been measured with scanning tunneling microscopy (STM) and transmission electron microscopy (TEM). Due to small size of the nanotubes and constant thermal motion of the carbon atoms they have interesting physical properties.

Carbon nanotubes can be prepared by laser vaporization of a carbon target in a tube furnace at $1200{ }^{\circ} \mathrm{C}$. Cobalt and nickel catalyst help the growth of the nanotubes. By use of two laser pulses $50 \mathrm{~ns}$ apart, growth conditions can be maintained over a large volume and for a long time. Flowing argon gas sweeps the nanotubes from the furnace to a watercooled copper collector just outside of the furnace. The result is single wall carbon nanotubes. Recently the carbon-arc method was developed to grow similar arrays of single-wall nanotubes. In this case nanotubes are produced from an ionized carbon plasma, and the heat from the discharge generated by the plasma. At present many 
international collaborations are working on measuring the properties of single-wall nanotubes. The scanning electron microscopy images of these nanotubes shows ropes of diameters between 10 to $20 \mathrm{~nm}$ and lengths of $100 \mu \mathrm{m}$. These ropes were examined in a TEM, each rope was found to consist of a bundle of single-wall carbon nanotubes aligned along a single direction. The unique electronic properties of nanotubes result from quantum confinement of electrons within the nanotube. Electrons can only propagate along the nanotube axis and their wave vectors point in this direction. The resulting number of one-dimensional conduction and valence bands effectively depends on the standing waves that are set up around the circumference of the nanotube. These simple ideas can be used to calculate the dispersion relations of the one-dimensional bands. Calculation of energy dispersion shows that one-third of small nanotubes are metallic and the rest are semiconducting. As the diameter of the nanotube increases there are more wave vectors allowed, thus the band gap decreases. Experiment shows that nanotubes of these different types are stable in pairs. So a metallic carbon nanotube, with semiconducting nanotubes surrounding it can behave like a conducting wire with an insulating shield.

Some of the interesting one-dimensional quantum effects of carbon nanotubes have been observed in Raman spectra, where lattice vibrations are introduced in the material by phonons through the inelastic scattering of light. Dispersion relations have been calculated and shows different vibrational modes coming from carbon atoms; as the nanotube diameter increases more phonon modes appear. Graphite, carbon fibers and carbon nanotubes are expected to be very strong and to resist fracture under extension. Thomas Ebbeson estimated Young's modulus of a carbon nanotube by measuring the 
vibrations of the free end of a nanotube that was clamped at the other end. Their estimates were consistent with the values of Young's modulus already measured for a graphene sheet. Efforts applying CNTs in electronic devices have been done, for example carbon nanotube field-effect transistors (CNFET) (Fig.3 \&4); CNFET performs with

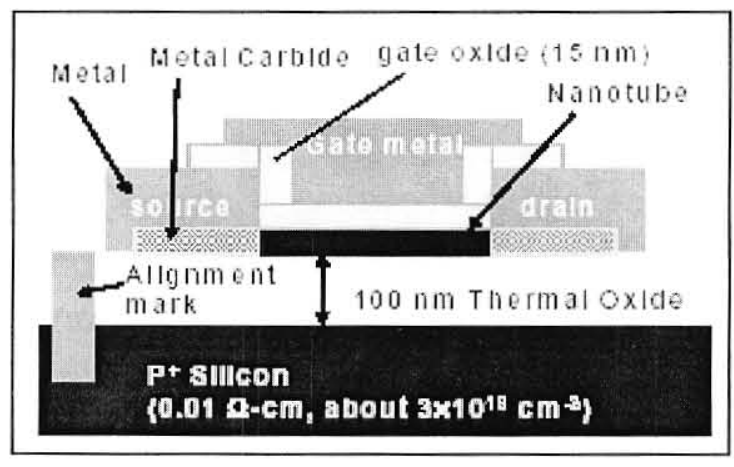

Fig.3 Schematic diagrams of the modeled, single wall carbon field effect transistor[8]

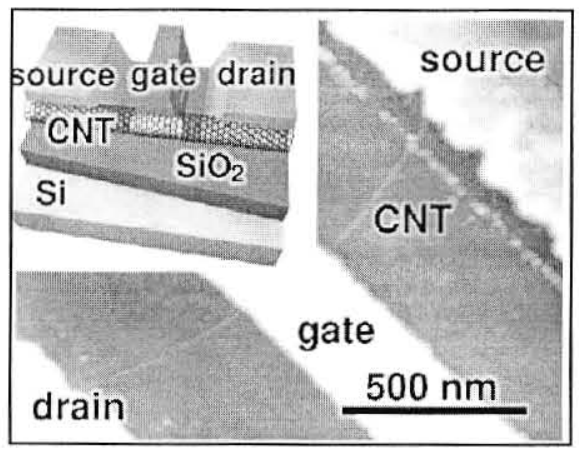

Fig.4 AFM image of CNT in a carbon-nanotube field-effect transistor (CNTFET).

bridging $1 \mu \mathrm{m}$ gape between source and drain [9]

comparable characteristics of silicon field-effect transistors. CNFETs are Schottky barrier transistors [5]. A composite of SWNT and Polyvinylcarbazole can create a light emitting 
diode [6]. Research on carbon nanotubes is one of the most popular fields at present and scientists are still characterizing CNTs and trying to use them to make devices. Carbon nanotubes are produced by catalyst driven synthesis by different methods; like arc discharge, Chemical Vapor Deposition (CVD), Pulse Laser Vaporization (PLV) and microwave plasma Chemical Vapor Deposition (PECVD). Catalyst samples prepared in different ways depend on catalyst support, the growth method and on the $\mathrm{pH}$ of the solution. Cobalt and iron were found to be essential in formation of acetylene and other carbon compounds. Iron leads to higher yield and cobalt enhances the quality of CNTs. The resulting CNTs can be SWNTs and MWNTs, grown as bundles or individually [10]. There are reports of carbon nanotubes made from silica, alumina, silicon and metals in a non-crystal structures; they are synthesized by using carbon nanotubes and porous membranes as templates, or by thin-film rolling. These nanotubes, however, are either amorphous or polycrystalline. There are reports about other types of nanotubes. For example single-crystal $\mathrm{GaN}$ nanotubes have been synthesized using hexagonal $\mathrm{ZnO}$ nanowires.

\section{Nanowires (NW)}

Development of nanotechnology and nanoscience has introduced new types of nanostructures called nanowires. NWs appear as semiconductors, carbides, nitrides, oxides, metals and metal oxides. For example GaAs, GaN, GaP, Si and Ge nanowires are semiconductor nanosize wire structures, while $\mathrm{GeO}_{2}, \mathrm{SiO}_{2}$ and $\mathrm{Ga}_{2} \mathrm{O}_{3}$ produce oxide NWs and gold, silver and other type of metals make metallic nanowires. Due to their small structures they have different properties than material in bulk and have many 
possible applications. Semiconductor nanowires, like GaAs can be used in FET or metallic NW can be used as tips for scanning tunneling microscopy. Various novel methods have been employed to create NWs.

Scientists have proposed three different approaches for NW growth; Vapor Liquid Solid (VLS), Vapor-Solid (VS), and Solution-Liquid-Solid (SLS) mechanisms. The principle of VLS growth is that the catalyst particle participating in the growth changes to liquid and the vapor phase atoms of the NW-base material saturates the liquid phase (Fig.5). Thus the temperature should not be less than the melting point of the catalyst. Nanowires can grow without a catalyst, for example, in $\mathrm{GeO}_{2}$ nanowire growth. In Vapor-Solid epitaxy the vapor atoms adsorbed to the initial crystalline whisker are only temporarily adsorbed and then diffuse along the lateral surface and reach the tip of the wire to continue the growth. If the vapor atoms don't diffuse and get to the tip they evaporate again.
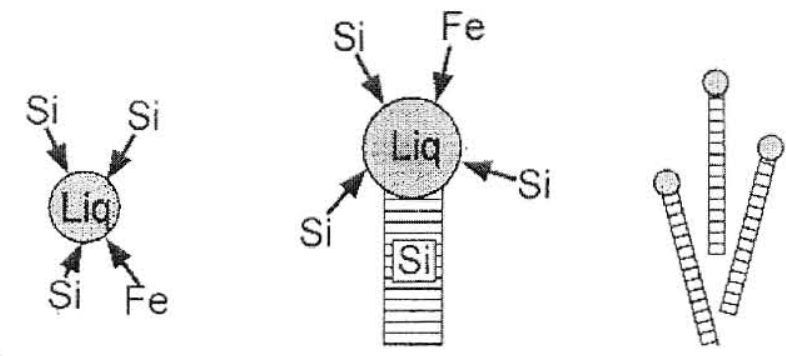

Fig.5 VLS mechanism of $\mathrm{Si}$ Nanowire synthesizes using Fe as catalyst nanoparticle

There are other ways to synthesize nanowires at lower temperatures ( around $200^{\circ} \mathrm{C}$ ). An example is the Solution-Liquid-Solid (SLS) method. In this electrochemical approach, a solution containing the nanowire constituent is used with the catalyst on the electrode. 
The difference between SLS and VLS is, instead of a being in the vapor phase, the atoms for nanowires growth contains in the chemical solution. The catalyst remains at the tip of the wire.

Chemical vapor deposition (CVD) applied for thin film growth has been extended to nanowire synthesis. Many types of nanowires have been grown by CVD, for example Si, Ge, GaAs and InP. The growth mechanism can be VLS or VS. In VLS growth the catalyst is attached to the tip of the wire.

Another method for nanowires synthesis is Pulsed Laser Vaporization (PLV). In this technique a hot oven, a pulse laser and a vacuum system are used. This technique is also called Laser Assisted Growth ( $L A G$ ). The pulsed laser can be either a Nd:Yag, $\mathrm{CO}_{2}$ or an excimer laser to vaporize the target and produce a plume while the growth mechanism can be VLS or VS. The catalyst can be on the target or on the substrate. The laser beam provides two dominant effects in laser ablation; collisional sputtering and thermal sputtering. Collisional sputtering employs the momentum transfer from the incident laser beam to the target material and thermal sputtering is the evaporation of the target by reaching a temperature higher than the melting point of the target.

Vapor Phase Evaporation has been used to synthesize metal and oxide whiskers and then extended to nanowire synthesis. The nanostructures are prepared by physical sublimation of a source material or through reduction of a variety of volatile metal halides. For example $\mathrm{Si}, \mathrm{Ge}, \mathrm{GeO}_{2}, \mathrm{Ga}_{2} \mathrm{O}_{3}$, $\mathrm{GaSe}$ can be synthesized using vapor phase evaporation. The reactor gets heated up and the evaporated or reduced atoms transport in the vapor phase toward the lower temperature gradient and nucleation happen in a random thermal 
or concentration fluctuation. In Tablel the growth of some nanowires is summarized (there are more possible growth methods for some of these nanowires).

Electrochemical Deposition or Template Approach is a production method of metallic nanowires synthesis. The synthesis is based on electrochemical deposition on a porous membrane (e.g. polycarbonate membrane). The pores of the membrane get filled with the nanowires so the next step is lift-off and etching of the membrane to release the nanowires [12].

Another remarkable method for nanowires synthesis is Oxide Assisted Growth (OAG). It is a VLS growth using no metal catalyst. Oxygen plays an important role in nucleation and growth of nanowires. The OAG technique allows large quantities of pure nanowires to be produced [13].

\begin{tabular}{|c|c|c|c|c|c|}
\hline \multirow{2}{*}{$\begin{array}{c}\text { Types } \\
\text { of }\end{array}$} & Source Materials (at\%) & Temp & Atmosphere & Pressure(Torr) & $\begin{array}{c}\text { Average } \\
\text { D of } \\
\text { NWs }\end{array}$ \\
\cline { 2 - 6 } & $\mathrm{Si}(95)+\mathrm{Fe}(5)$ & 1200 & $\mathrm{Ar}$ & 100 & $\sim 12$ \\
\hline & $\mathrm{Zn}(50)+\mathrm{Se}(50)$ & 800 & $\mathrm{Ar}+\mathrm{H}_{2}$ & 200 & 60 \\
\hline $\mathrm{SiO} 2$ & $\mathrm{Si}(10)+\mathrm{SiO}(85)+\mathrm{Fe}$ & 1250 & $\mathrm{Ar}$ & 150 & $20 \sim 90$ \\
\hline & $\mathrm{Ge}+\mathrm{Fe}$ & 900 & $\mathrm{Ar}$ & 150 & $15 \sim 80$ \\
\hline & $\mathrm{Ga}+\mathrm{SiO}$ & 350 & $\mathrm{Ar}+\mathrm{H}_{2}$ & 100 & 60 \\
\hline $\mathrm{ZnO}$ & $\mathrm{Zn}+\mathrm{Se}$ & 200 & $\mathrm{Ar}+\mathrm{H}_{2}$ & 200 & 40 \\
\hline
\end{tabular}

Table. 1 
The nanowire diameter ranged from $5 \mathrm{~nm}$ to $500 \mathrm{~nm}$ depending on the material and the length varies between $1 \mu \mathrm{m}$ to $100 \mu \mathrm{m}$. The morphology of NWs can be crystalline, poly crystalline and amorphous. Semiconductor nanowires can be made from one element, for example from group IV (Si, Ge) or it can be a multi compound, for example binary group III-V (GaAs, GaP, InAs and InP), binary group II-VI (ZnS, ZnSe, CdS and CdSe) on ternary III-V (GaAs/P, InAs/P) [14]. Superlattice nanostructures in the form of heterostructures have been synthesized from group III-V and group VI in a uniform, crystalline structure.

The results of the VLS growth process for synthesizing varieties of semiconductor nanowires depend on the Pseudobinary phase diagram of the reactant chemicals (Fig.6). The percentage of the catalyst to the material is thereby defined. For example for GaAs laser assisted growth using $\mathrm{Au}$ as the catalyst, it is revealed that the atomic percentage of GaAs to $\mathrm{Au}$ should be $(\mathrm{GaAs})_{0.95} \mathrm{Au}_{0.05}$. Also, phase diagram helps to approximately determine the synthesis temperature range.

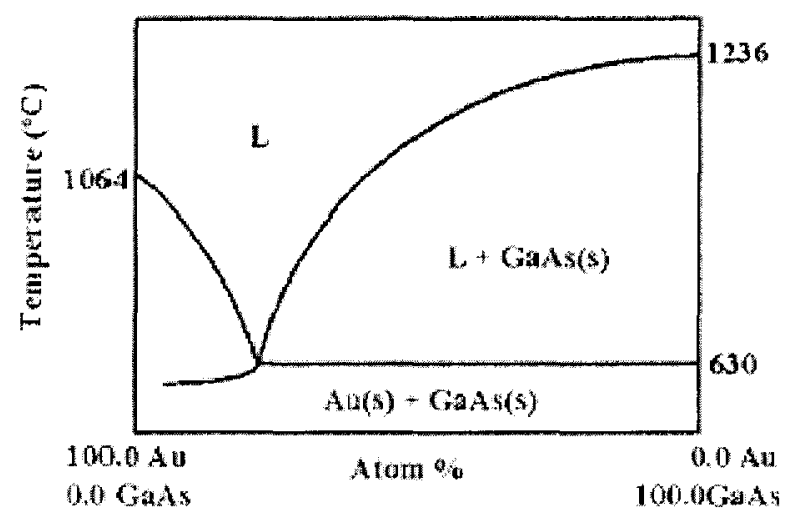

Fig. 6 Pseudobinary phase diagram for $\mathrm{Au}$ and $\mathrm{Ga}$ As 
The resulting NWs synthesized by the LAG method can be as small as $3 \mathrm{~nm}$ in diameter and about $10 \mu \mathrm{m}$ long. High resolution microscopic images from a SEM or TEM reveal that the catalyst particle is attached to the tip of the NW and Scanning Tunneling Microscopy (STM) or Field Effect TEM (FETEM) show the crystalline structure of the nanowires and the uniformity of the atoms in the structure. (Fig.7)

Super-lattice nanowires are essentially heterostructures of two types of semiconducting crystalline structures. Composition of modulated superlattices of $\mathrm{GaAs} / \mathrm{GaP}$ nanowires
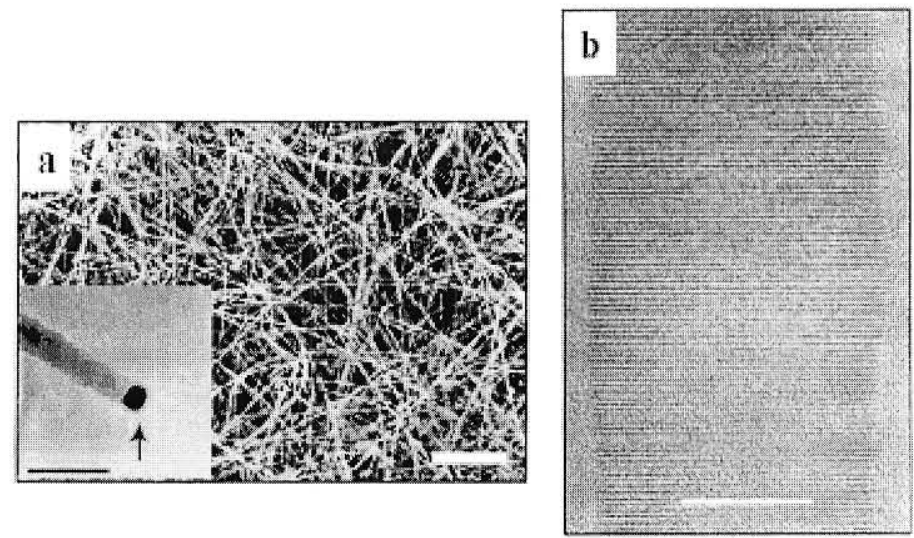

Fig.7 (a) Low resolution TEM image of GaAs Nanowires, the catalyst particle is attached to the tip of the NW, (b) FETEM image of the crystalline structure of GaAs Nanowire growth by VLS method

have been synthesized (Fig.8). Furthermore, In As/InP, n-Si/p-Si nanowires and n-InP/pInP have been synthesized. The growth happens by alternating the vaporization between two different materials and changing the vapor-phase reactant material in a specific period of time. These novel structures composed of nanowires can have unique photonic 
and electronic properties and the potential applications includes nanosize LEDs and nano-barcode.
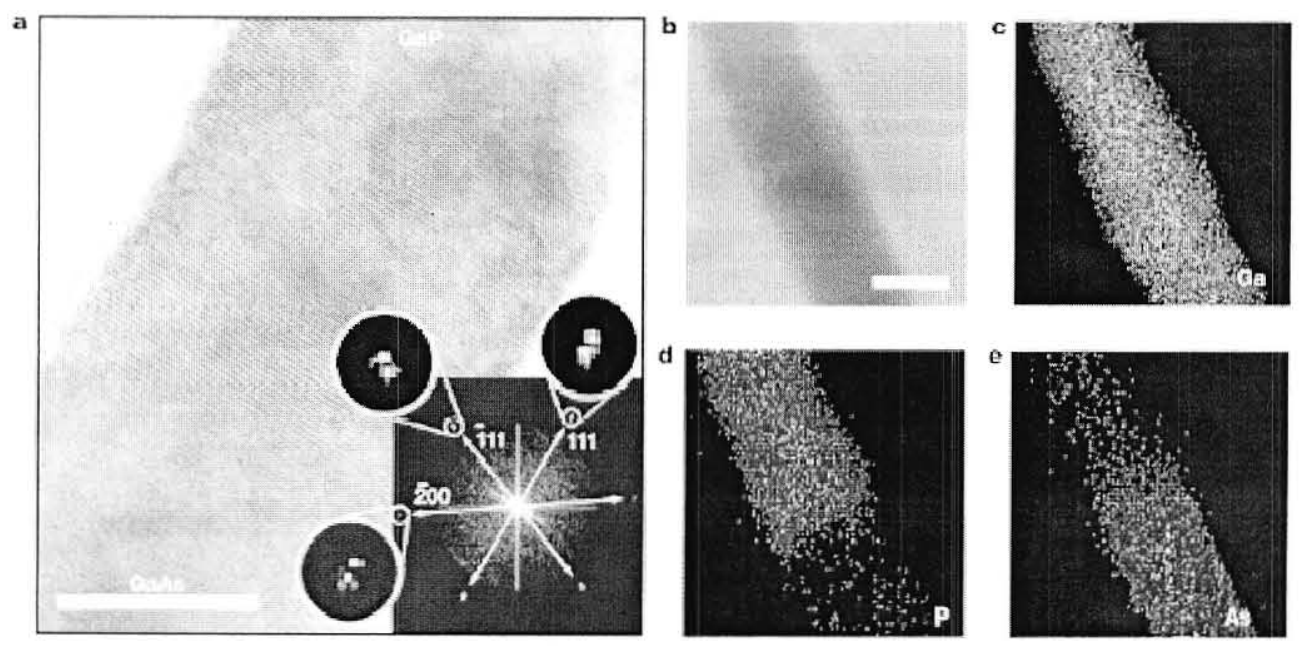

Fig.8 (a) GaAs/GaP nanowire TEM image and the diffraction pattern (b),(c),(d) and ( e) EDS result for NW , the border of the chemicals is very clear and sharp

There are other types of nanostructures such as nanoribbons, nanorods and nanobelts, which are subgroups of nanowires and we will be covering later in chapter III.

Carbon-based materials (diamond and in-plane graphite) display the highest measured thermal conductivity of any known material at moderate temperatures.[1] The discovery of carbon nanotubes in 1991 (Ref. 2) has led to speculation[3] that this new class of onedimensional carbon could have a thermal conductivity equal to or greater than that of diamond and graphite. Past measurement of the thermal conductivity of nanotubes has been limited by the low quality of available samples. Recent advances[4,5] in nanotube synthesis have made possible the growth of high-purity crystalline bundles of nearly monodisperse single-walled carbon nanotubes (SWNT's). Such materials are well suited 
to transport studies, including measurements of electrical and thermal conductivity. In this work we report measurements of the thermal conductivity of single-walled carbon nanotubes from $300 \mathrm{~K}$ to $8 \mathrm{~K}$. We are motivated to measure the thermal conductivity of SWNT's to test the speculation that nanotubes have an exceptionally high thermal conductivity, as well as to probe the phonon structure in these unique materials. 


\section{CHAPTER II}

\section{A. Introduction}

Many experimental methods have been developed over the past centuries to measure the fundamental thermal properties of materials. One important class among them, the is called $3 \omega$ method, uses a narrow-band detection technique and therefore gives a relatively better signal-to-noise ratio. In this method, either the specimen itself serves as a heater and at the same time a temperature sensor, if it is electrically conductive and with a temperature-dependent electric resistance, or for electrically nonconductive specimen, a metal strip is artificially deposited on its surface to serve both as the heater and the sensor. Feeding an ac electric current of the form $I 0 \sin \omega t$ into the specimen or the metal strip creates a temperature fluctuation on it at the frequency $2 \omega$, and accordingly a resistance fluctuation at $2 \omega$. This further leads to a voltage fluctuation at $3 \omega$ across the specimen. Corbinol is probably the first to notice that the temperature fluctuation of an ac heated wire gives useful information about the thermal properties of the constituent material. Systematic investigations of the $3 \omega$ method were carried out mainly during the 1960 's and in the last ten years, which made the method practical. However, in the previous studies the heat conduction equation was solved under the approximations either only for the high frequency limit or only for the low frequency limit. With those approximations one lost either the information on the thermal conductivity or the information on the specific heat of the specimen. 
The thermal conductivity in thin-film structures has received particular attention in recent years in both fundamental and applied research. The thermal properties of these low-dimensional structures are basically involved in several applications, such as in small-scale thermoelectric devices or electronic, and optoelectronic devices. Understanding the heat diffusion mechanisms is becoming increasingly important for improving their performance and reliability. Therefore, the thermal conductivity is critical information.

Measurements of thermal properties, specific heat, and thermal conductivity of condensed matter constitute one of the major characterization of physical properties of condensed matter. Measurements of thermal conductivity of a solid, for example, offers a very nice way to investigate the elementary excitations, which participate as heat carriers or limit their mean free path in given system. It also allows one to study mechanisms, such as defects in a crystal, which limit the mean free path of the heat carrying excitations. On the other hand, specific heat measurements allows one to directly monitor the free energy change of a given system as the external parameter such us temperature varies. This unique ability of specific heat measurements follows from the fact that specific heat per unit volume $\mathrm{Cp}$ is defined thermodynamically as

$$
\mathrm{Cp}=1 / \mathrm{V}^{*}(\mathrm{dH} / \mathrm{dT})=\mathrm{T} / \mathrm{V}(\mathrm{dS} / \mathrm{dT})
$$

Where T,H,S,V are the temperature, the enthalpy, the entropy, and the volume of a given system, respectively. 
In addition to the above, more traditional usage of the thermal measurements, one cane use specific heat measurements as a probe to the dynamics of system, since specific heat is expressed as the enthalpy fluctuation of the system from the statistical mechanical point of view. Using the linear response theory, it is fairly straightforward to define the dynamic, i.e., frequency-dependent specific heat $\mathrm{Cp}(\omega)$ in terms of the time correlation function of the enthalpy of the system, namely,

$$
C_{p}(w)=C_{p_{0}}+\frac{i w}{k_{b} T^{2} V} \int_{0}^{\infty} \mathrm{e}^{i \omega t} \delta(\mathrm{H}(0)) \delta(\mathrm{H}(t)) d t
$$

where $k_{b}$ is the Boltzmann constant, $C_{p_{0}}$ is the usual static specific heat, and the enthalpy fluctuation of the system is $\delta(\mathrm{H}(t))=\mathrm{H}(t)-\{H\}$

Although it is not common to actually observe the frequency-dependent specific heat, there are experimental situations where these dynamic specific heats were indeed measured. For example, Smith observed the frequency-dependent specific heat in germanium and Smith and Holland discussed it in terms of a delay in equilibrium. Frequency-dependent specific heat was also measured near the glass transition of glycerol and potassium-calcium nitrite mixture.

We have adopted the third harmonic detection method, an ac modulation method using the heater as a sensor simultaneously. The third harmonic detection method was originally discovered by Corbino long ago[7] and exploited extensively by Smith. More recently Birge and Nagel and Jung used a planar heater in applying the same method to probe the sow dynamics associated with the glass transition, while Cahill[8] utilized a 
thin line heater to measure thermal conductivity of solids. In my thesis I shall call the calorimetric technique based on the third harmonic detection method a $3 \omega$ technique.

\section{B. Theoretical basis of $3 \omega$ technique}

In order to measure the temperature oscillation of a heater itself we use the aforementioned third harmonic detection method, which is briefly described here. If one drives the heater with a current at frequency $\omega I(t)=I 0 \cos (\omega t)$, then one gets Joule heating at frequency $2 \omega$ at the heater and thus the temperature of the heater oscillates at the same frequency with a complex amplitude $\delta$ Tout-of-phase. Since the heater is made of metal such as gold, its temperature coefficient of resistivity, $\alpha=(1 / \mathrm{R})(d \mathrm{R} / d \mathrm{~T})$, is not zero and as a result its resistance also oscillates at frequency $2 \omega$. Thus $R(t)=R_{0}\left[1+\alpha\left|\delta T_{\text {out-of-phas }}\right| \cos (2 \omega t+\varphi)\right]$, where $\varphi$ represents the phase shift of $\delta T_{\text {out-of-phas }}$ with respect o the power oscillations.

Due to the driving current $I(t)$ through the heater, the voltage across the heater appears as

$$
\begin{aligned}
V(t) & =I(t) R(t)=I 0 \cos \omega t \cdot R_{0}\left[1+\alpha\left|\delta T_{\text {out-of-phas }}\right| \cos (2 \omega t+\varphi)\right] \\
& =I_{0} R_{0} \cos \omega t+1 / 2 I_{0} R_{0} \alpha\left|\delta T_{\text {out-of-phas }}\right| \cos (\omega t+\varphi)+1 / 2 I_{0} R_{0} \alpha\left|\delta T_{\text {out-of-phas }}\right| \cos (3 \omega t+\varphi)
\end{aligned}
$$

It is the second and third terms that contains the thermal information of the sample we want to measure. 


\section{The 1D heat conduction equation and its solution}

We consider a uniform rod- or filament-like specimen in a four-probe configuration as for electrical resistance measurement (Fig. 9). The two outside probes are used for feeding an electric current, and the two inside ones for measuring the voltage across the specimen. Differing from being a pure electrical resistance measurement, however, here it requires that

i) the specimen in between the two voltage probes be suspended to allow the temperature fluctuation,

ii) all the probes have to be highly thermal conductive, to heat sink the specimen at these points to the sapphire substrate, and

iii) the specimen has to be maintained in a high vacuum and the whole setup be heat shielded to the substrate temperature to minimize the radial heat loss through gas convection and radiation.

In such a configuration and with an ac electrical current of the form $I_{0} \sin \omega t$ passing through the specimen, the heat generation and diffusion along the specimen can be described by the following partial differential equation and the initial and boundary conditions:

$$
\begin{aligned}
& \rho C p \frac{\partial}{\partial t} T(x)-k \frac{\partial^{2}}{\partial \alpha^{2}} T(x,) \\
& \quad=\frac{I_{0}^{2} \sin ^{2} \omega t}{I S}\left[R+R^{\prime}\left(T(x)-T_{0}\right)\right] .
\end{aligned}
$$



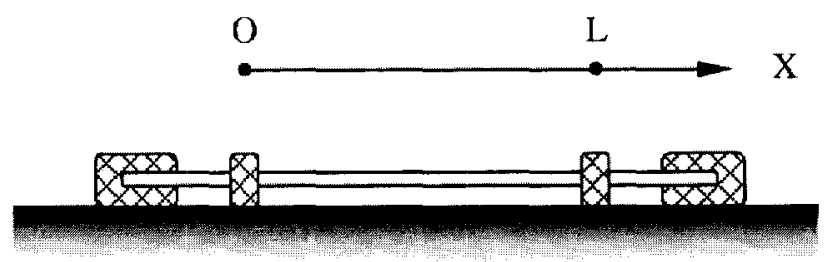

Fig. 9. Illustration of the four-probe configuration for measuring the specific heat and thermal conductivity of a rodor filament-like specimen is shown. The specimen is heat sunk to the sapphire substrate through the four electric contacts, but the part in between the two voltage contacts needs to be suspended, to allow the temperature variation. A high vacuum is needed and a thermal shielding is preferred to eliminate the radial heat current from the specimen to the environment.

$$
\begin{aligned}
& T(O)=T_{0} \\
& T(I)=T_{0} \\
& T(x,-x)=T_{0}
\end{aligned}
$$

where $C p, \mathrm{k}, R$, and rare the specific heat, thermal conductivity, electric resistance and mass density of the specimen at the substrate temperature $T_{0}$, respectively. $R^{\prime}=(d R / d T) T_{0} . L$ is the length of the specimen between voltage contacts, and $S$ the cross section of the specimen. We have assumed that the electric current was turned on at $t=-$ infinity.

Let $\Delta(x, t)$ denote the temperature variation from $T 0$, i.e., $\Delta(x, t)=T(x, t)-T_{0}$, Eqs. (3) and (4) then become

$$
\begin{aligned}
& \frac{\partial}{\partial r} \Delta(x+r) \alpha \frac{\partial-}{\partial x^{2}} \Delta(x+)-c \sin ^{2} \text { or } \Delta(x, t)=b \sin ^{2} \omega r \\
& \Delta(0 r)=0 \\
& \Delta(L, r)=0 \\
& \Delta(x,-\alpha)=0
\end{aligned}
$$

where $\alpha=\mathrm{k} / \rho C p$ is the thermal diffusivity, and $b=I_{0}^{2} R \quad / \rho C p L S$, and $c=I R^{\prime} / \rho C p L S$ 
Using the impulse theorem, $\Delta(x, t)$ can be represented as the integral of the responses of the specimen to the instant "force" $b \sin 2 \omega t$ at each time interval:

$$
\left.\Delta(x, t)=\int_{-\infty}^{t}-1 x, t\right) d t
$$

where $z(x, t ; \tau)$ satisfies

$$
\begin{aligned}
& \frac{\partial z}{\partial}-\alpha \frac{\partial^{2}-}{m^{2}}-c \sin ^{2} \omega t z=0 \text {, } \\
& =10, t)=0 \\
& =|L, I|=0 \\
& =(x, \tau+0)=b \sin ^{2} \omega \tau \text {. }
\end{aligned}
$$

$z(x, t ; \tau)$ can be expanded in the Fourier series:

$$
z(x, t)=\sum_{n=1}^{\infty} l_{n}(t ;-) \sin \frac{n \pi x}{L}
$$

Substituting Eq. (10) into Eq. (8), we have

$$
\sum_{n=1}^{n}\left[\frac{d L_{n}}{d t}+\left(\frac{n^{2}}{\gamma}-c \sin ^{2} \omega t\right) \tau_{n}\right] \sin \frac{n \pi x}{L}=0
$$

where $\gamma=\mathrm{L}^{2} / \pi^{2} \alpha$ is the characteristic thermal time constant of the specimen for the axial thermal process.

The term $c \sin 2 \omega t$ can be neglected if $\left.\mathrm{n}^{2} / \gamma\right\rangle \mathrm{c}$, or equivalently

$$
\frac{I_{0}^{2} R^{\prime} L}{V^{-} \pi^{2} k S}
$$

Condition (12) means that the heating power inhomogeneity caused by resistance fluctuation along the specimen should be much less than the total heat power. This condition is usually held. For example, in a typical measurement one could have

$$
I_{0}=10 \mathrm{~mA} . \quad R^{\prime}=0.1 \Omega \mathrm{K} \quad I=1 \mathrm{~mm},
$$


$\mathrm{S}=1 / 10^{2} \mathrm{~mm}^{2}$, and $\mathrm{k}=100 \mathrm{~W} / \mathrm{m} \mathrm{K}$, the left-hand side of Eq.(12) is then about $1 / 10^{3}$ even for the $n=1$ case.

After dropped off the $c \sin 2 \omega t$ term, the solution of the ordinary differential Eq. (11) is

$$
Y_{n}(t)=C_{n}(\tau) e^{-\left(n^{2}(y) t r\right)}
$$

where $C n(\tau)$ can be determined using the initial condition in Eq. (9), together with the relation

$$
\begin{aligned}
& \Sigma_{n=1 / 2}^{*}\left[1-(-1)^{n}\right] n \pi \sin n \pi L=1 \text { for } 0 \times x<L \\
& S R=\frac{R^{\prime}}{L} \int_{0}^{I}\left[T(x+1) T_{0}\right] d x . \\
& C_{n}(r)=\frac{2 b\left[1-(-1)^{n}\right]}{n \pi} \sin ^{2} \omega r .
\end{aligned}
$$

Using Eqs. (13) and (14), Eq. (10) becomes

$$
-(x+\pi)=\sum_{n=1}^{n} \sin \frac{n \pi n}{I} \frac{2 b\left[1-(-1)^{n}\right]}{n \pi} \sin ^{2} \omega \tau e^{-\left(n^{2} \gamma(r-\tau)\right.}
$$

Substituting Eq. (15) into Eq. (7) and remembering that $\Delta(x, t)=T(x, t)-T 0$, we obtain the temperature distribution along the specimen:

$$
T(x, t)-T_{0}=\Delta \sum_{n=1}^{\infty} \frac{\left[1-1-1 !^{n}\right]}{2 n^{3}} \times \sin \frac{n \pi x}{L}\left[1-\frac{\sin \left(2 \omega t+\phi_{n}\right)}{1+\cot ^{2} \phi_{n}}\right]
$$


where $\cot \Phi n=2 \omega \gamma / n^{2}$, and $\Delta 0=2 \gamma b / \pi=2(I 0)^{2} R /(\pi \mathrm{kS} / L)$ is the maximum de temperature accumulation at the center of the specimen. $\Delta 0$ is only $\mathrm{k}$ dependent. The information of $C p$ is included in the fluctuation amplitude of the temperature around the dc accumulation.

Figure 10 illustrates how the amplitude of such a temperature fluctuation depends on the frequency of the electric current.[9] The amplitude reaches the maximum as $\omega \gamma \rightarrow 0$, i.e., when the thermal wavelength $\lambda \gg \nu$ (where $\lambda$ is defined as $\lambda=\sqrt{ }(\alpha / 2 \omega)$ ). But it shrinks to zero along the line of the averaged temperature accumulation when $\omega \gamma>>1(\lambda<<L)$. The temperature fluctuation will result in a resistance fluctuation, which can be calculated as

$$
S R=\frac{R^{\prime}}{L} \int_{0}^{I}\left[T \mid x+1-T_{0}\right] d x
$$

Using Eq. (16) and the relation $1 \sin n-L) i x=[1-(-1) \mid \Sigma n \pi$, the resistance fluctuation can be expressed as

$$
D R=R^{\prime}+\sum \frac{|1-1-1|^{2}}{2 \pi n^{2}}\left[1-\frac{\sin 2 \omega^{*}+\phi_{n !}}{1+\cot ^{2} \phi_{n}}\right]
$$

As a product of the total resistance $R+\delta R$ and the current $I 0 \sin \omega t$, the voltage across the specimen contains a $3 \omega$ component $V_{3} \omega(t)$. Obviously, the $n=2$ term in $V_{3} \omega(t)$ automatically vanishes. If only taking the $n=1 \mathrm{term}$, which introduces a relative error of the order $3 \cdots$ at low frequencies, we have

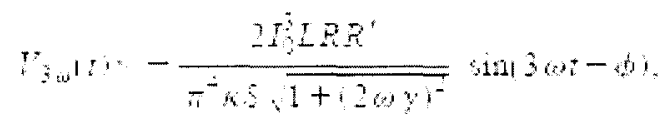




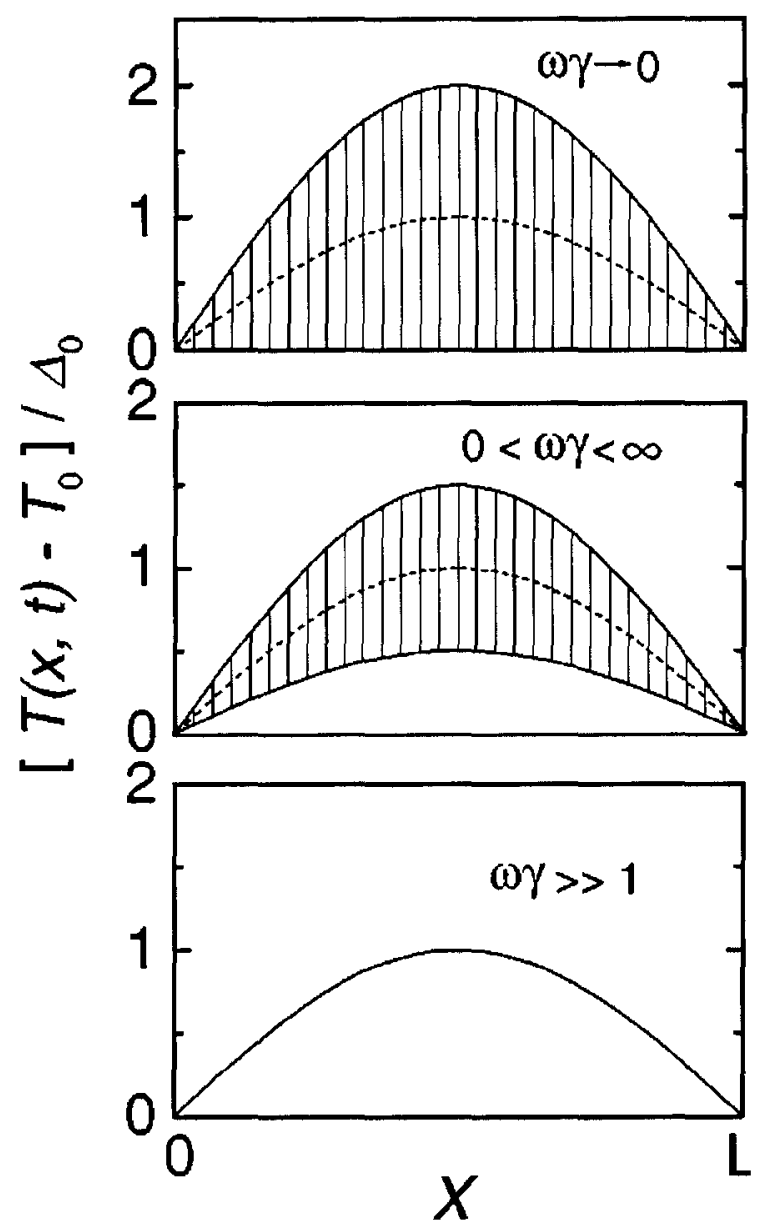

Fig.10 : Temperature fluctuation along the specimen under a driving ac current $I_{0} \sin \omega t$ is shown. The fluctuation amplitude is marked as shadowed area. It reaches the maximum at the limit $\omega \gamma \rightarrow 0$, and shrinks to a line as $\omega \gamma \rightarrow \infty$. The line in the middle of the fluctuation range denotes the $\mathrm{dc}$ temperature accumulation along the specimen, which reaches the maximum value of $\Delta 0$ (defined in the text) at the center of the specimen.[9]

where we have redefined the phase constant $\varphi=\pi / 2-\varphi 1$, so that

$$
\tan 4,20 y
$$

If using the root-mean-square (rms) values of voltage and current as what the lock-in amplifier gives, Eq. (19) Becomes (hereafter we always let $V 3 \omega$ denotes the rms value of $V_{3}(t)(t)$, and $I$ denotes the rms value of $\left.I_{0} \sin \omega t\right)$;

$$
r_{3 \omega} \frac{4 I^{3} I R R^{\prime}}{\pi^{2}+\sqrt{1+12 \omega y^{2}}} .
$$


By fitting the experimental data to this formula, we can get the thermal conductivity $\kappa$ and thermal time constant $\gamma$ of the specimen. The specific heat can then be calculated as

$$
c_{g}=\pi^{2} y k p^{2}
$$

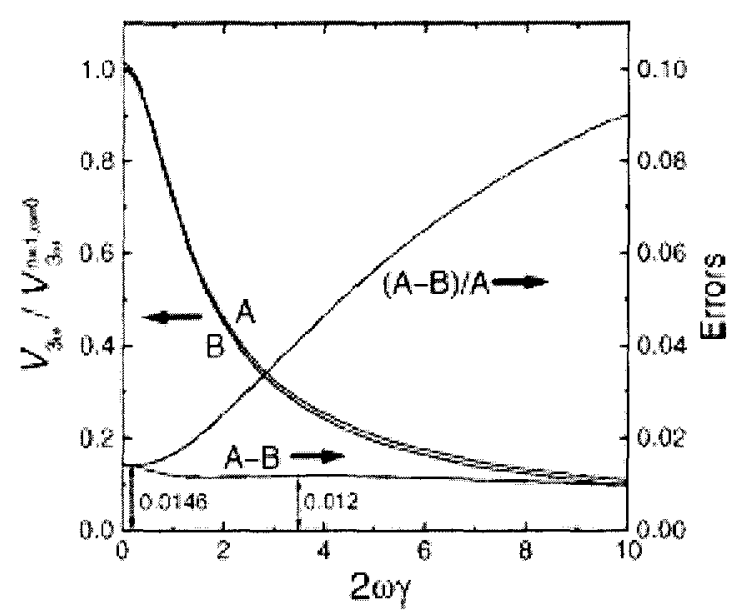

Fig.11 The errors of $V / 3 w$ caused by truncating the $n>1$ terms in Eq. (18) are shown. Curve A represents the exact solution of the $3 \omega$ voltage amplitude. Curve $\mathrm{B}$ is the $3 \omega$ voltage of the $n=1$ term alone. The difference between them is nearly a constant at low frequencies, plotted as curve $\mathrm{A}-\mathrm{B}$. The relative error of $V / 3 \omega$ increases with $\omega$, illustrated as curve $(\mathrm{A}-\mathrm{B}) / \mathrm{A}$.[9]

The following alternative form makes it more clear how the $3 \omega$ voltage depends on the dimensions of the specimen:

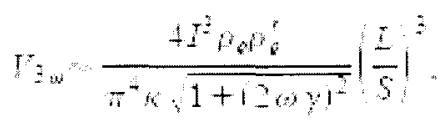

where $\rho_{\mathrm{e}}$ is the electrical resistivity of the specimen, $\rho_{\mathrm{e}}{ }^{r} \equiv\left(\mathrm{d} \rho_{\mathrm{e}} / \mathrm{dT}\right)$. 


\section{The high and low frequency limits}

Sometimes the measurement has to be performed at the low frequency limit $\omega \gamma \rightarrow 0$ $(\lambda>>L)$, e.g., when the specimen is extremely thin and long. In this case, $V_{3 \omega}$ is nearly frequency independent. To an accuracy of roughly $3^{-4}$ it takes the form

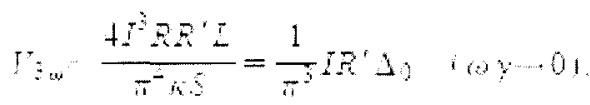

If the measurement is performed at the low frequency limit, one can only get the thermal conductivity of the specimen, but loses the information on specific heat, as in Cahill's treatment for a two-dimensional heat diffusion problem.

At the high frequency limit $\omega \gamma \rightarrow \infty(\lambda<<L)$, on the other hand, Eqs. (19)-(23) become quite inaccurate due to truncating the $n>1$ terms in Eq. (18). In this limit, all the $\varphi_{n}$ approach to zero, and the amplitude of the summation over the time-dependent terms in

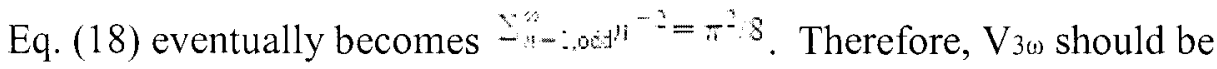

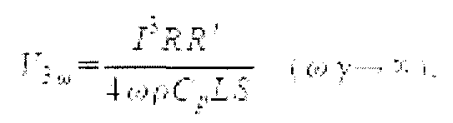

which is exactly the same as Holland's result. Simply truncating the $n>1$ terms at the $\omega \gamma$ $\rightarrow \infty$ limit will result in a coefficient of $2 / \pi^{2}$, instead of $1 / 4$, for $V 3 \omega$ in Eq. (25).

At the high frequency limit, one can only get the specific heat of the specimen, but loses the information on its thermal conductivity. 


\section{Error analysis}

The error of $V_{3 \omega}$ caused by truncating the $n>1$ terms in Eq. (18) is illustrated in Fig. 3. Curve $\mathrm{A}$ is the normalized fluctuation amplitude of Eq. (18) containing terms up to $n=9$, taken from a numerically generated time sequence. It almost represents the exact solution. Curve B is the fluctuation amplitude of the first term alone. It appears that the difference between A and B (shown as curve A-B in Fig. 11) is nearly a constant in the frequency range $0<2 \omega \gamma<10$. It approaches to $\sum_{n=3.00 e^{n}}^{\eta^{-4}} \sim 0.014$ as $\omega \rightarrow 0$. However, because $V 3 \omega$ decreases with frequency, the relative error of $V_{3 \omega}$ increases with $\omega$ (illustrated as curve (A-B)/A in Fig. 11).

The relative error of $\tan f$ in Eq. (20) should also increase with frequency. Indeed, the experimental data of $\tan \Phi$ curve away from linearity at high frequencies. By fitting the data to Eq. (20), the high frequency inaccurate side of Eq. (20) provides more weight on the slope, so that one will get a noticeably smaller $\gamma$ than the true value.

The case of using Eq. (21) is fortunately just the opposite. The amplitude of $V_{3 \omega}$ is relatively large at the low frequency side where Eq. (21) is very accurate. If we fit curve to Eq. (21) in the frequency range $0<2 \omega \gamma<4$, the obtained $\mathrm{k}$ is only $3.5 \%$ higher, and $\gamma$ is $2 \%$ lower than the true values. $C p$ is then only $1.4 \%$ higher than the true value.

Because the error in Eq. (21) is nearly frequency independent at low frequencies (curve A-B in Fig. 11), it can be further and easily reduced by shifting the fitting curve upwards by a small amount, i.e., fitting the data to the following form:

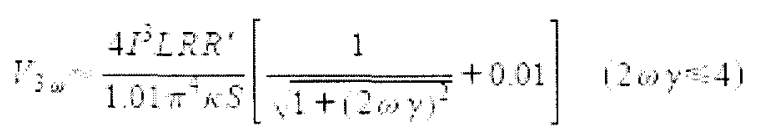


Fitting curve A to Eq.(26) in the frequency range $0<2 \omega \gamma<4$ yields $\mathrm{k}, \gamma$, and $C p$ that are all within $0.1 \%$ of their true values. In this case, the error introduced by truncating the $n<1$ terms becomes negligibly small comparison with errors of other sources, such as from the size estimation.

If one truncates the $n>1$ terms in Eq. (16) to calculate the temperature fluctuation, the error will be more significant than truncating the $n>1$ terms in Eq. (18). This is because the summation converges as $n^{-3}$ in Eq. (16), not as $n^{-+}$in Eq. (18).

\section{Redial heat loss}

In the previous discussion, we have neglected the radial heat loss through radiation. Such heat loss per unit length from a cylindrical rod of diameter $D$ to the environment of temperature $T_{0}$ can be expressed as:

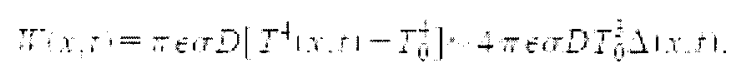

where $\sigma=5.67 * 10^{-3} \mathrm{~W} / \mathrm{m}^{2}\left(\mathrm{~K}^{2}\right)^{2}$ is the Stefan-Boltzmann constant, and $\varepsilon$ is the emissivity. Considering such heat loss, Eqs. (5) and (6) can be rewritten as

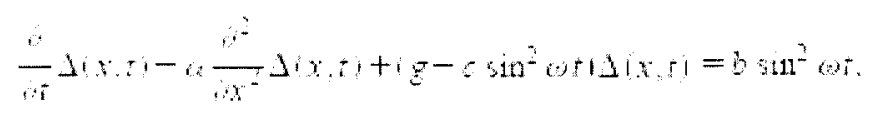


LII $1=0$

$\Delta|x-x|=0$

where $g=16 e^{-T} T_{0}^{3} p \mathrm{D}$ Equation (11) then becomes

$$
\sum\left[\frac{d t}{d t}+\left(\frac{n^{2}}{y}+g-\operatorname{com}^{2} \omega t\right) U_{n}\right] \sin \frac{n \pi x}{I}=0
$$

Now if we truncate the $n>1$ terms again and replace the factor $1 / \gamma+g$ with $1 / \gamma_{\text {ap }}$, Eq. (30) will take the similar form as Eq. (11). The final approximation solution is therefore

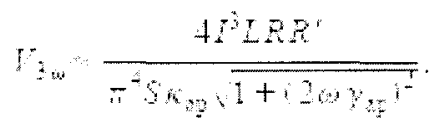

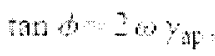

where kap $=(1+\gamma g)^{*} \mathrm{k}$ is the apparent thermal conductivity, and $\gamma_{\mathrm{ap}}=\gamma /(1+\mathrm{g} \gamma)$ is the apparent thermal time constant of the specimen. The apparent dc temperature accumulation is $\Delta_{y}=\Delta_{0} n+g y$ at the center of the specimen.

Obviously, radiation heat loss can be neglected if

$$
g y x
$$

For a cylindrical rod, condition (33) becomes $16 \operatorname{tar} T_{1}=\pi^{2}, 0$, which means that the radiation power inhomogeneity caused by the temperature fluctuation along the specimen should be much less than the axial heat current or the total heating power.

Condition (33) is usually held for measurements performed below room temperature. For example, if one has a specimen of the size $L=1 \mathrm{~mm}$ and $D=10^{-2} \mathrm{~mm}$, and assuming $\mathrm{k}=100 \mathrm{~W} / \mathrm{m} \mathrm{K}, \quad T_{0}=300 \mathrm{~K}$, the product $g \gamma$ is only around $2.5^{*} 10^{-3}$ even if using the emissivity of a black body. 
However, for specimens of significantly longer or thinner, or if the measurement is performed at significantly higher temperatures, condition (33) will be violated. In these cases, the apparent thermal conductivity is larger than the actual value by an amount due to the radial heat loss, for cylindrical rod which is

$$
\left.\mu_{n g}=k 1+g\right)=k+\frac{16 e \pi T^{3} I^{2}}{\pi^{2} D} .
$$

If one knows the emissivity, then both $\mathrm{k}$ and $C p$ of the specimen can be calculated. Otherwise if the emissivity is unknown, one will lose the information of $\mathrm{k}$. Nevertheless, one can still get $C p$ of the specimen. The reason is, by substituting kap and $\gamma_{\text {ap }}$ into Eq. (22) as if there is no radial heat loss, the $(1+g \gamma)$ factors in kap and in $\gamma_{\text {ap }}$ just cancels out, which yields the correct value of $C p$ :

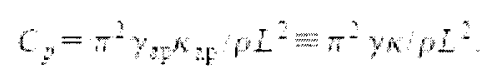

Although this analysis is made for cylindrical rod, the conclusions are also revelatory for specimens of other shapes. One can easily deduce the factor $g \gamma$ for particular specimens if needed.

Another kind of radial heat loss, the heat loss through gas convection, also introduces a linear-term correction to the heat conduction equation. The final solution is therefore the same as Eqs. (31) and (32) except that now $g=4, \ldots, C_{y} D$ the cylindrical specimen of diameter $D$ (where $\eta$ is the surface thermal conductivity). Similar to the case of radiation heat loss, one needs to know $\eta$ before being able to calculate $\mathrm{k}$. But one can still obtain $C p$ of the specimen through Eq. (35) without knowing $\eta$. This has been proven to be true 
experimentally, even when the heat loss through gas convection is much larger than the axial thermal current

For eliminating the heat loss through gas convection, one simply needs a high vacuum. For eliminating radiation heat loss, however, simply using a radiation shielding at the substrate temperature $T_{0}$ would be useless because it is the radiation power inhomogeneity along the specimen that matters. Nevertheless, we feel that a simple heat shielding at $T_{0}$ will at least help minimize the static radial heat current from the specimen to the environment, especially for measurements performed above room temperature. Otherwise, such heat current could cause the temperature of the specimen to be inaccurate and the whole heat conduction processes becomes complicated. 


\section{CHAPTER III}

\section{Experiment}

\section{Thin films}

There are a number of different techniques that facilitate the deposition or formation of very thin films (of the order of micrometers, or less) of different materials on a silicon wafer (or other suitable substrate). These films can then be patterned using photolithographic techniques and suitable etching techniques. Common materials include silicon dioxide (oxide), silicon nitride (nitride), polycrystalline silicon (polysilicon or poly), and aluminium.

A number of other materials can be deposited as thin films, including noble metals such as gold. Noble metals will contaminate microelectronic circuitry causing it to fail, so any silicon wafers with noble metals on them have to be processed using equipment specially set aside for the purpose. Noble metal films are often patterned by a method known as "lift off", rather than wet or dry etching.

Often, photoresist is not tough enough to withstand the etching required. In such cases a thin film of a tougher material (eg, oxide or nitride) is deposited and patterned using photolithography. The oxide / nitride then acts as an etch mask during the etching of the underlying material. When the underlying material has been fully etched the masking layer is stripped away. 


\section{Photolithography}

Photolithography is the basic technique used to define the shape of micromachined structures in the three techniques outlined below. The technique is essentially the same as that used in the microelectronics industry, which will be described here.

Figure 12a shows a thin film of some material (eg, silicon dioxide) on a substrate of some other material (eg, a silicon wafer). It is desired that some of the silicon dioxide (oxide) is selectively removed so that it only remains in particular areas on the silicon wafer (figure $12 f)$.

Firstly a mask is produced. This will typically be a chromium pattern on a glass plate. The wafer is then coated with a polymer which is sensitive to ultraviolet light (figure $12 b$ ), called a photoresist. Ultraviolet light is then shone through the mask onto the photoresist (figure 12c). The photoresist is then developed which transfers the pattern on the mask to the photoresist layer (figure 12d).

There are two types of photoresist, termed positive and negative. Where the ultraviolet light strikes the positive resist it weakens the polymer, so that when the image is developed the resist is washed away where the light struck it - transferring a positive image of the mask to the resist layer. The opposite occurs with negative resist. Where the ultraviolet light strikes negative resist it strengthens the polymer, so when developed the resist that was not exposed to ultraviolet light is washed away - a negative image of the mask is transferred to the resist.

A chemical (or some other method) is then used to remove the oxide where it is exposed through the openings in the resist (figure 12e). Finally the resist is removed leaving the patterned oxide (figure 1f). 
(a)

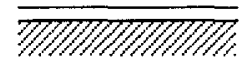

(b)
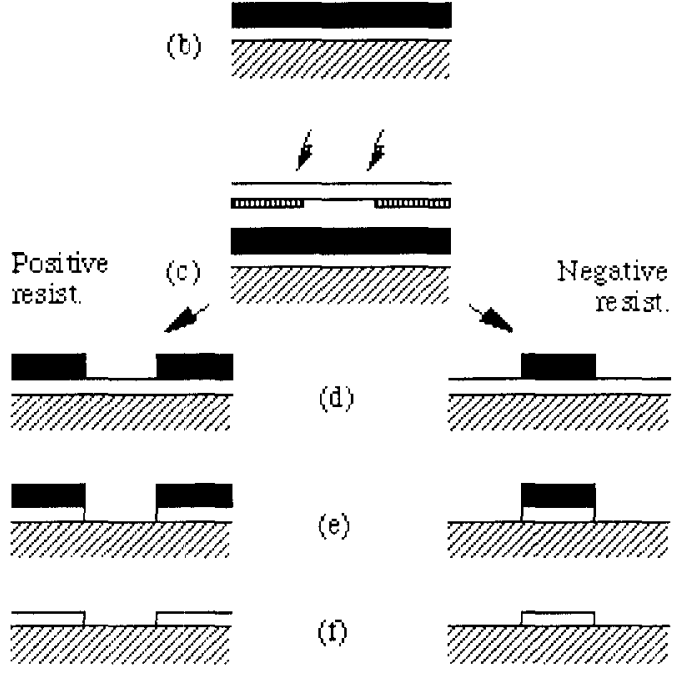

Figure 12.

\section{Wet etching}

Wet etching is a blanket name that covers the removal of material by immersing the wafer in a liquid bath of the chemical etchant. Wet etchants fall into two broad categories; isotropic etchants and anisotropic etchants.

Isotropic etchants attack the material being etched at the same rate in all directions. Anisotropic etchants attack the silicon wafer at different rates in different directions, and so there is more control of the shapes produced. Some etchants attack silicon at different rates depending on the concentration of the impurities in the silicon (concentration dependent etching).

Isotropic etchants are available for oxide, nitride, aluminium, polysilicon, gold, and silicon. Since isotropic etchants attack the material at the same rate in all directions, they remove material horizontally under the etch mask (undercutting) at the same rate as they etch through the material. This is illustrated for a thin film of oxide on a silicon wafer in 
figure 13, using an etchant that etches the oxide faster than the underlying silicon (eg, hydroflouric acid).

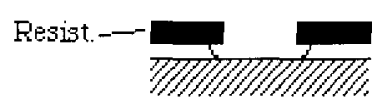

Figure13.

This illustrates the isotropic wet etching of a thin film of material. The photoresist is black, and the substrate grey stripes. The film is etched through, and the etching continues to further under-cut the mask.

Anisotropic etchants are available which etch different crystal planes in silicon at different rates. The most popular anisotropic etchant is potassium hydroxide $(\mathrm{KOH})$, since it is the safest to use.

Silicon wafers are slices that have been cut from a large ingot of silicon that was grown from a single seed crystal. The silicon atoms are all arranged in a crystalline structure, so the wafer is monocrystalline silicon (as opposed to polycrystalline silicon mentioned above). When purchasing silicon wafers it is possible to specify that they have been sliced with the surface parallel to a particular crystal plane.

The simplest structures that can be formed using $\mathrm{KOH}$ to etch a silicon wafer with the most common crystal orientation (100) are shown in figure 14. These are V shaped groves, or pits with right angled corners and sloping side walls. Using wafers with different crystal orientations can produce grooves or pits with vertical walls. 


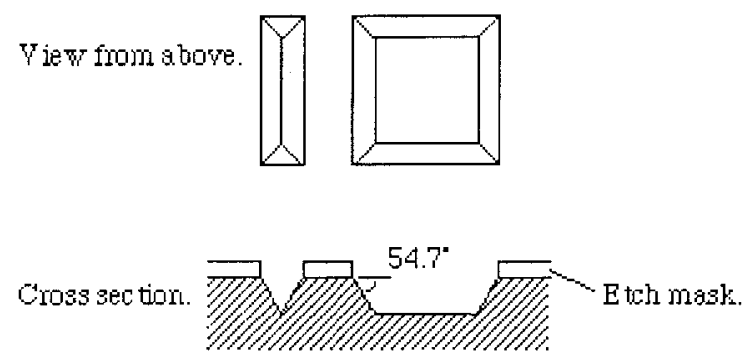

Figure 14.

Both oxide and nitride etch slowly in $\mathrm{KOH}$. Oxide can be used as an etch mask for short periods in the $\mathrm{KOH}$ etch bath (ie, for shallow grooves and pits). For long periods, nitride is a better etch mask as it etches more slowly in the $\mathrm{KOH}$.

Concentration Dependent Etching. High levels of boron in silicon will reduce the rate at which it is etched in $\mathrm{KOH}$ by several orders of magnitude, effectively stopping the etching of the boron rich silicon.

The boron impurities are usually introduced into the silicon by a process known as diffusion. A thick oxide mask is formed over the silicon wafer and patterned to expose the surface of the silicon wafer where the boron is to be introduced (figure 15a). The wafer is then placed in a furnace in contact with a boron diffusion source. Over a period of time boron atoms migrate into the silicon wafer. Once the boron diffusion is completed, the oxide mask is stripped off (figure 15b).

A second mask may then be deposited and patterned (figure 15c) before the wafer is immersed in the $\mathrm{KOH}$ etch bath. The $\mathrm{KOH}$ etches the silicon that is not protected by the mask, and etches around the boron doped silicon (figure 15d). 


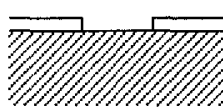

(a)
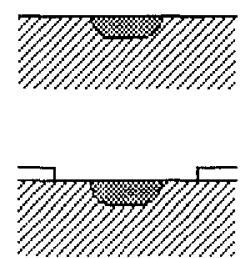

(c)

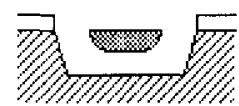

(d)

Figure 15.

Boron can be driven into the silicon as far as $20 \mu \mathrm{m}$ over periods of 15 to 20 hours, however it is desirable to keep the time in the furnace as short as possible. With complex designs, etching the wafer from the front in $\mathrm{KOH}$ may cause problems where slow etching crystal planes prevent it from etching beneath the boron doped silicon. In such cases the wafer can be etched from the back, however this is not without disadvantages (longer etching times, more expensive wafers, etc). The high concentration of boron required means that microelectronic circuitry cannot be fabricated directly on the boron doped structure.

\section{Dry etching}

The most common form of dry etching for micromachining applications is reactive ion etching (RIE). Ions are accelerated towards the material to be etched, and the etching reaction is enhanced in the direction of travel of the ion. RIE is an anisotropic etching technique. Deep trenches and pits (up to ten or a few tens of microns) of arbitrary shape and with vertical walls can be etched in a variety of materials including silicon, oxide and 
nitride. Unlike anisotropic wet etching, RIE is not limited by the crystal planes in the silicon.

\section{Lift off}

Lift off is a stencilling technique often used to pattern noble metal films. There are a number of different techniques, the one outlined here is an assisted lift off method.

A thin film of the assisting material (eg, oxide) is deposited. A layer of resist is put over this and patterned, as for photolithography, to expose the oxide in the pattern desired for the metal (figure 16a). The oxide is then wet etched so as to undercut the resist (figure 16b). The metal is then deposited on the wafer, typically by a process known as evaporation (figure 16c). The metal pattern is effectively stencilled through the gaps in the resist, which is then removed lifting off the unwanted metal with it (figure $16 \mathrm{~d}$ ). The assisting layer is then stripped off too, leaving the metal pattern alone (figure 16e).

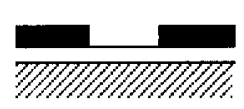

(a)

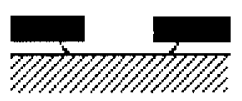

(b)

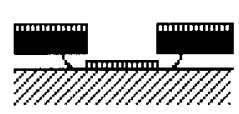

(c)
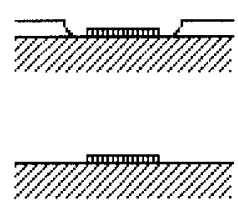

(e)

Figure 16.

There are lift off techniques in which only photoresist is used as the stencil. In the assisted lift off, an intermediate layer assists in the process to ensure a clean lift off and 
well defined metal pattern. When noble metals are used it is desirable to deposit a thin layer of a more active metal (eg, chrome) first, to ensure good adhesion of the noble metal.

\section{Mask design}

Many of the processes described in this thesis report involve some form of photolithography; which requires masks. The design of photolithography masks is generally fairly straightforward. Normally, designs incorporate relative large structures $(1-10 u m+)$ compared to the sub-micron structures that are now incorporated into advanced VLSI technologies. All that is required is some suitable CAD (computer aided design) software, and a platform to run it on.

\section{Software for mask design}

The basic CAD software required is a "layout editor". This enables you to place different polygons onto different layers - each layer being a mask design for a particular step in the fabrication process -, assemble these into "structures" (or "cells") which can be placed adjacent to one another on the final design, and export the design file in a suitable format. Many software packages will provide additional features. It is also possible to use some drawing packages, provided one can translate the files into a suitable format for mask fabrication. If you are familiar with ordinary CAD or drawing packages, then you will have few problems switching to a layout editor. For mask I used during this research Ledit editor. 
Fig 17. is showing a mask which was developed by L-edit.
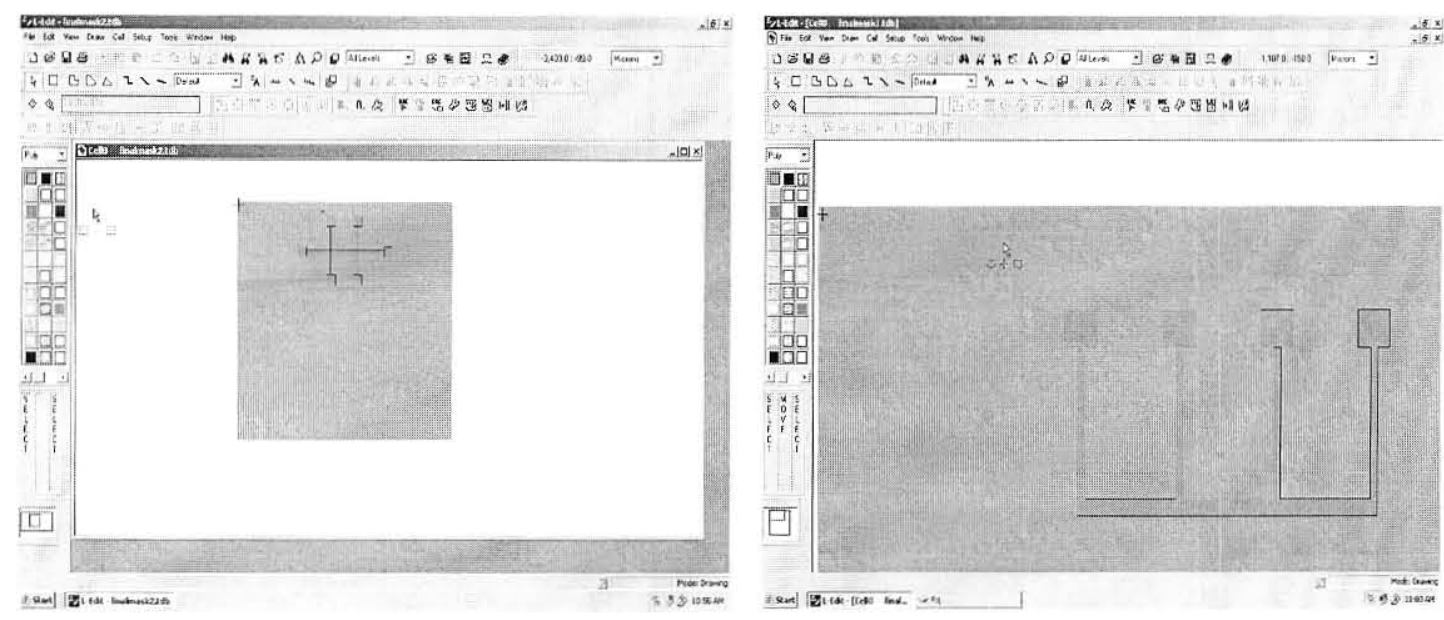

Figure 17.

\section{Alignment marks}

In order to line one layer up with a previously fabricated layer, when performing photolithography, it is necessary to incorporate appropriate marks into your mask design to facilitate this. The foundry producing the devices will probably have specific marks that they would like you to use, and these may need to be placed in a specific position on your design. They may also have additional requirements; such as the inclusion of a scribe lane around each chip, to indicate where the wafer is to be cut when it is diced, the inclusion of a unique mask number, and an indication of layer names on the mask so that it is possible to tell by looking how far through the fabrication process a wafer has progressed. See figure 18. 

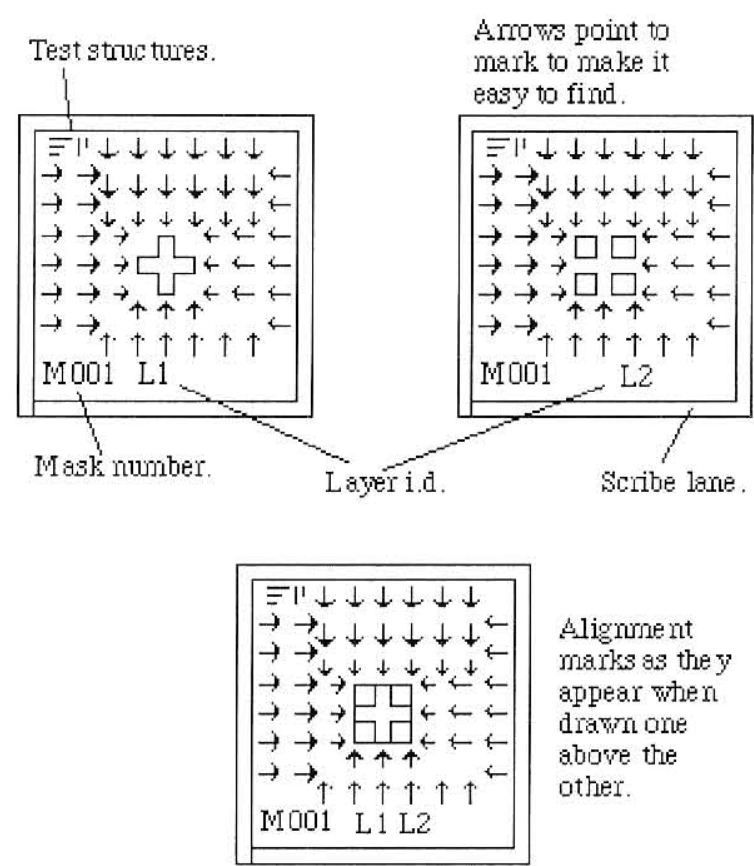

$\Delta$ very simple alignme nt design for two layers, (example only, not to sole)

Figure 18.

Figure 19 is showing an alignment marks that we used in this research work.

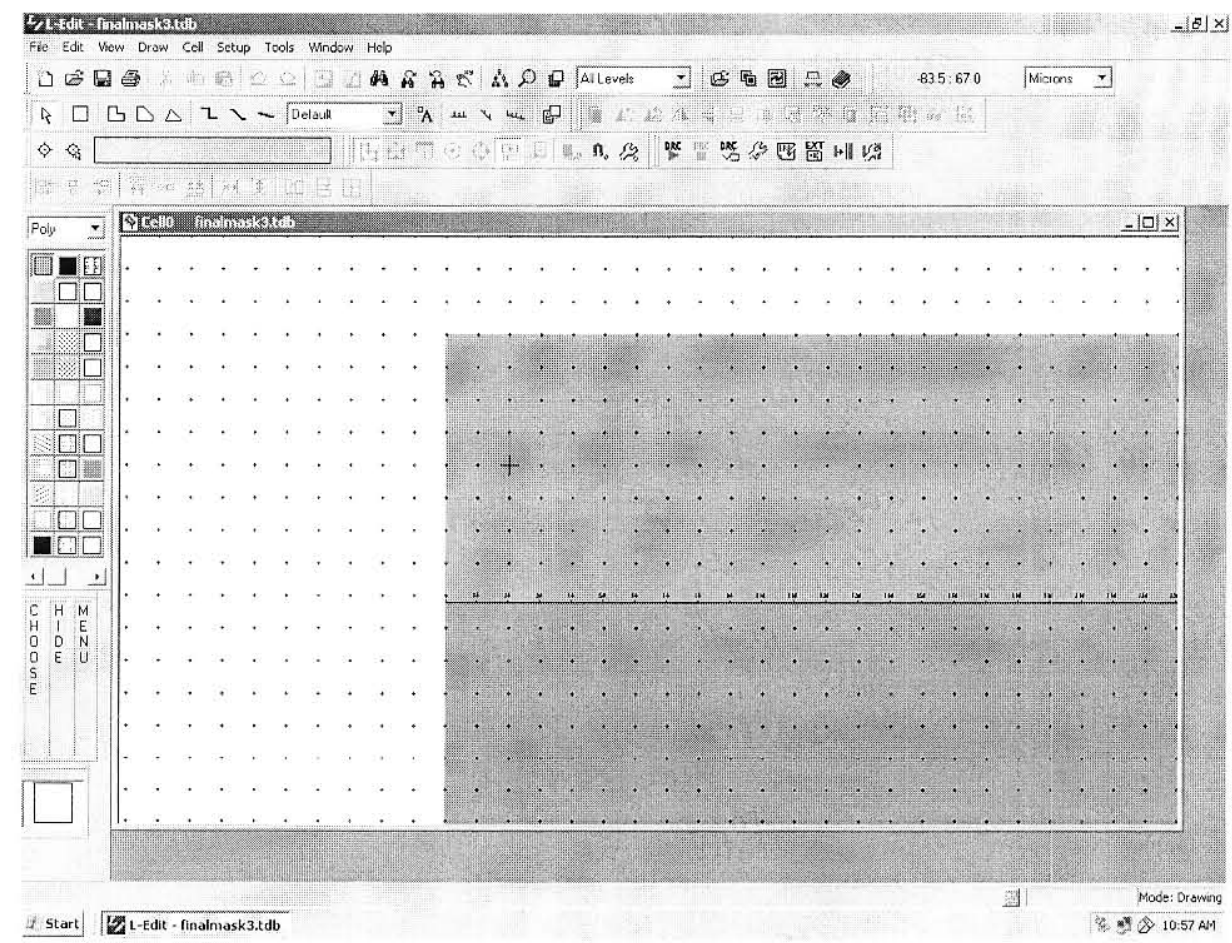

Figure 19. 


\section{Minimum feature size}

The concept of a "minimum feature size" is important in mask design. The minimum feature size is the width of the smallest line or gap that appears in your design. When the mask is produced, the minimum feature size will be required to set up the equipment. A small minimum feature size will mean that the mask creation process takes a long time and becomes increasingly expensive; with very small features (sub-micron) it may be necessary to perform additional processing of the mask for optical correction purposes. This should not happen for most micromachining applications, however: one would expect to have minimum feature sizes in the region of a few micrometers.

\section{Design rules}

Design rules are developed for each separate fabrication sequence. They include the minimum feature size, but essentially they tell you how much overlap you need to leave between two mask layers to ensure that the two features will be coincident when fabricated. They also ensure that there is a suitable gap between two features that should not touch when fabricated. Design rules depend upon the expected alignment error that will be introduced during fabrication. This will be comparatively large when doing double sided alignment. If we take an arbitrary value of $+/-1$ um alignment error, for example, and we wish to etch a via hole over a metal pad, then it would be wise to leave at least an underlap of at least 2 um (try and work in multiples of the minimum feature size, if possible) to ensure the final structure is fabricated as desired: see figure 20. 
The fabric ation sequence is the same for both (de posit and pattem metal layer first, then an insulating layer).
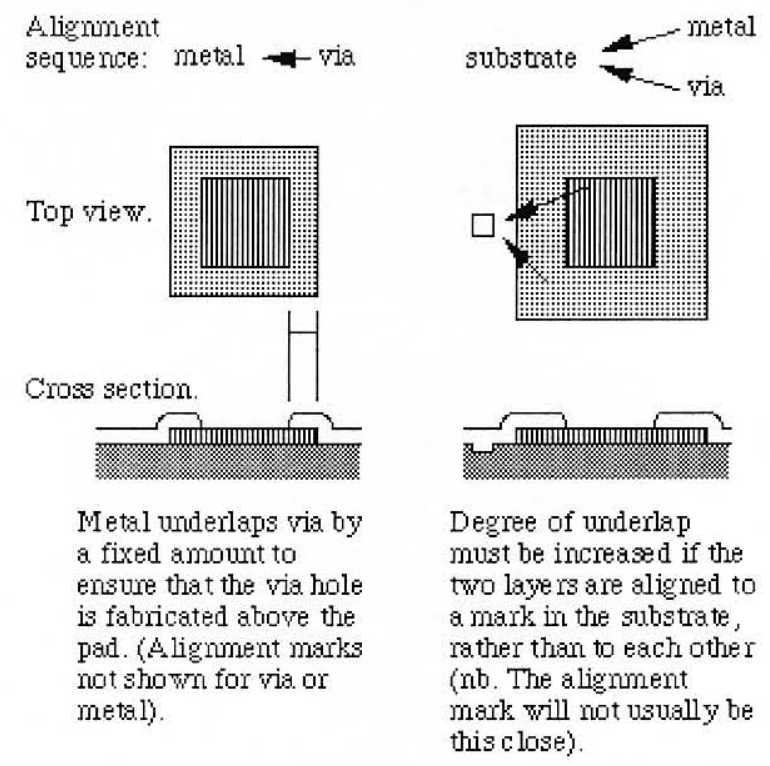

Figure 20.

Also illustrated in figure 20 is the consequence of alignment sequence, which may be different to fabrication sequence. Alignment errors will be cumulative. For instance, if the metal pattern is aligned to a mark etched into the substrate, and the via pattern is aligned to this same mark, then the metal pattern could end up -1 um out of alignment and the via pattern +1 um: an error of 2 um between metal and via pattern. Here you would want to try and leave at least 4um underlap if possible. Note that if the via were to be aligned to a mark on the metal pattern, it would only be $+/-1$ um out of registration with the metal, but $+/$ - 2 um out of alignment with the mark on the substrate.

Many mask design software packages come with design rule checkers, which will automatically check your design against the rules that you enter, and highlight any deviation from the rules. 


\section{Experiment}

We have tested this $3 \omega$ method on two kinds of specimens: silicon/silicon dioxide and quartz/quartz with single-wall carbon nanotubes, by just using the approximation solution (21). The electrical resistance of the former specimen has a positive temperature coefficient, and the latter; a negative one. Within appropriate ranges of frequency and $r_{3,} \times r^{2}$ and $r_{3 w} \times 1 \overline{+(26 y)^{2}}$. current, we do find.

Figure 21 shows the block diagram for the measurement. A digital lock-in amplifier such as a SR810 made by Stanford Research Inc. was selected. All the filters on the lock-in were turned off, and the dc coupled input mode was selected, to ensure the observation of a true frequency dependence of $V_{3 \omega}$. Before measuring the $3 \omega$ signal, the phase of the lock-in amplifier was adjusted to zero according to the $1 \omega$ voltage component. The phase angle of $V_{3 \omega}$ is then $-\varphi$ if $R \times 180^{\circ}-1 R^{\prime}>0$ according to Eq. (19). We used a simple electronic circuit (the lower panel of Fig. 21) to convert the $1 \omega$ sine wave voltage from the sine out of the lock in to an ac current, and then we fed the current into the specimen. Because the $3 \omega$ voltage signal is deeply buried in the $1 \omega$ voltage signal, a certain amount of dynamic reservation is required for the lock in if, in order to keep the simplicity of this method, one is not using a bridge circuit to cancel out the $1 \omega$ signal. We kept the dynamic reservation unchanged relative to the total magnification of the lock in during the entire measurement. 


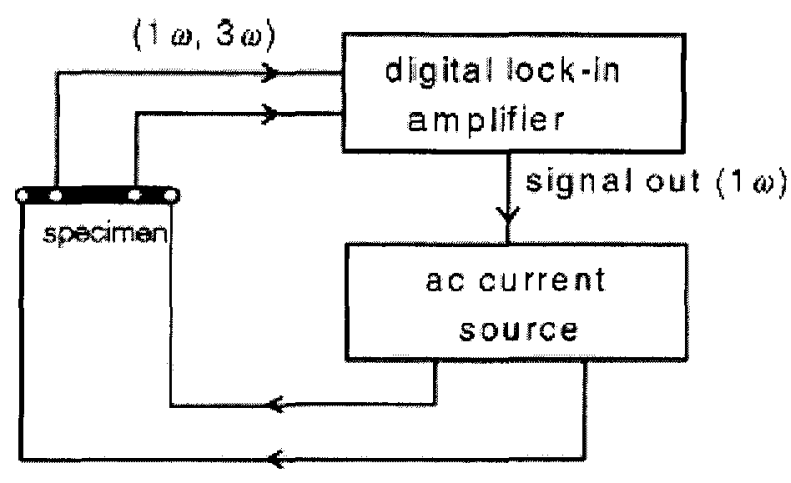

SR810 lock-in amplifier

Figure 21

There are two ways to perform the measurement. In the first, the substrate of the specimen is maintained at fixed temperatures, then the frequency dependence of $V_{30}$ is measured. In this way, we can check the $I^{3}$ and the $1, \overline{1+(2 \omega y)^{2}}$ dependencies of $V_{3(\omega)}$ as well as the relation $\tan \varphi=2 \omega \gamma$.

Because $V_{3 \omega} \sim I^{3}$, one will get a much larger signal by using a larger $I$. However, there are three reasons for not using a very large $I$. First, it is required by condition (12). Second, radiation heat loss will be significant when the temperature modulation is large, as condition (33) indicates. Third, excessive heat accumulation on the specimen would even create a considerably large temperature gradient at the silver paste contacts, which might violate the boundary condition in Eq. (4). In all the cases, the expected relations such as $V 3 \omega \sim I^{3}$ will not be held. On the other side, the relation will also be violated if $I$ is too small so that $V_{3 \omega}$ becomes comparable to, or even smaller than the spurious $3 \omega$ signals that come from the current or other sources. In our measurement, the total heating power was maintained such that the temperature modulation along the specimen was around 1 K. Nevertheless, if the $3 \omega$ voltage is too small to measure then one has to increase the current for creating a larger temperature fluctuation. In this case, the actual (averaged) 
temperature of the specimen has to be corrected afterwards by comparing the resistance of the specimen measured with the larger current and that measured with a much smaller one.

From Eq. (23), a longer and thinner specimen also gives a larger signal. However, a larger $L$ corresponds to a larger thermal time constant $\gamma\left(\gamma \sim L^{2}\right)$, and hence, a lower frequency window for measurement. In practice, it will be inconvenient to perform the measurement below $1 \mathrm{~Hz}$. A larger length and a smaller cross section or diameter could also violate the conditions (12) and (33), and thus violate the expected $I_{3}$ and the $1 \longdiv { 1 + 1 2 \omega y ) ^ { 2 } }$ dependencies of $V_{3 \omega}$.

In the second way of measurement, the temperature of the substrate is slowly ramped up or down at a fixed rate, meanwhile the working frequency of the lock-in amplifier is switched between a few set values. The maximum working frequency is adjusted by keeping $2 \omega \gamma<4$ (i.e., $\varphi<76^{\circ}$ according to Eq. (20)). And, the electric current is adjusted roughly to maintain a fixed dc temperature accumulation (i.e. $\sim 1 \mathrm{~K}$ ). The whole process, including the temperature ramping, parameters adjusting, and frequency switching, are all controlled by a personal computer.

The measurement of thermal conductivity is the prerequisite for simulation of thermal behavior of interconnection systems using low $\mathrm{k}$ dielectric( like silicon dioxide) and silicon and quartz also. Especially for low density and porous low k dielectric materials, the low thermal conductivity may cause heat dissipation problems. The so-called $3 \omega$ measurement technique (transient hot wire method) was established to determine the thermal conductivity of thin films. Measurements of standard substrates and films show validation of the achieved thermal conductivity values and agreement with published, 
commonly accepted values. The method was successfully applied to determine the thermal conductivity of porous low k dielectric materials using special test structures.

The name $3 \omega$ measurement technique derives from the method of detection; a current at angular frequency $\omega$ is caused to flow through the wire resulting in heating at frequency $2 \omega$ (dc heating is negligible). The voltage signal at $3 \omega$, measured with a lock-in amplifier, is proportional to the ac temperature in the specimen. By proper calibration, the thermal conductivity is obtained from the frequency dependence of the signal. The measured values are thermal coefficient of resistivity, heater resistance, temperature and the $\omega$ and $3 \omega$ signals. Heating power, the geometry (width, length, thickness of the heater and film) and material properties affect the measurement.

The $3 \omega$ method has been used extensively for measuring the thermal conductivity of thin films and bulk materials [1-4]. We applied it to determine the thermal behavior of porous ultra low $\mathrm{k}$ dielectric materials. The geometry (cross-section, layout) of the 4-pad test structure for the $3 \omega$ method is shown below (Fig. 22,Fig 23.). A substrate is covered with the film to be measured. On top the test structure is fabricated.

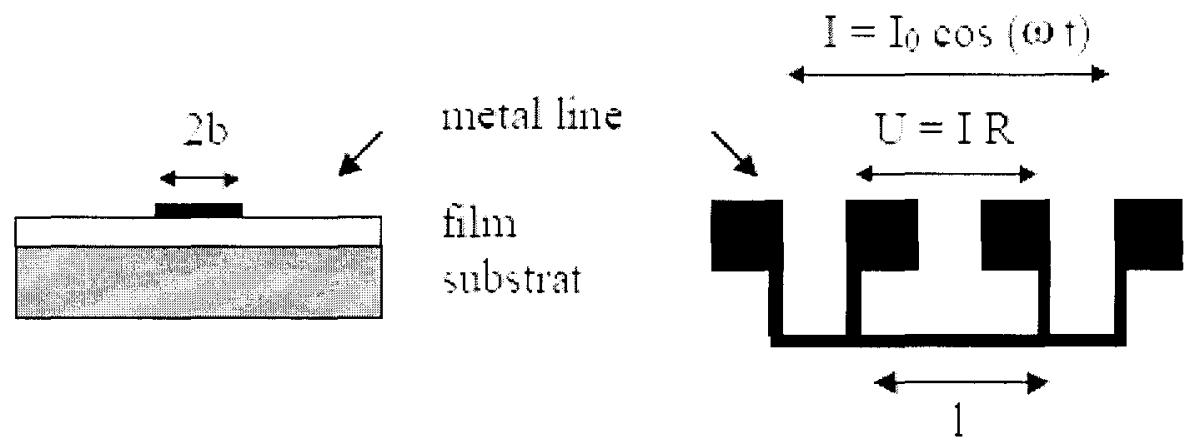

Fig. 22: Geometry of the 4 pad test structure for the $3 \omega$ method 


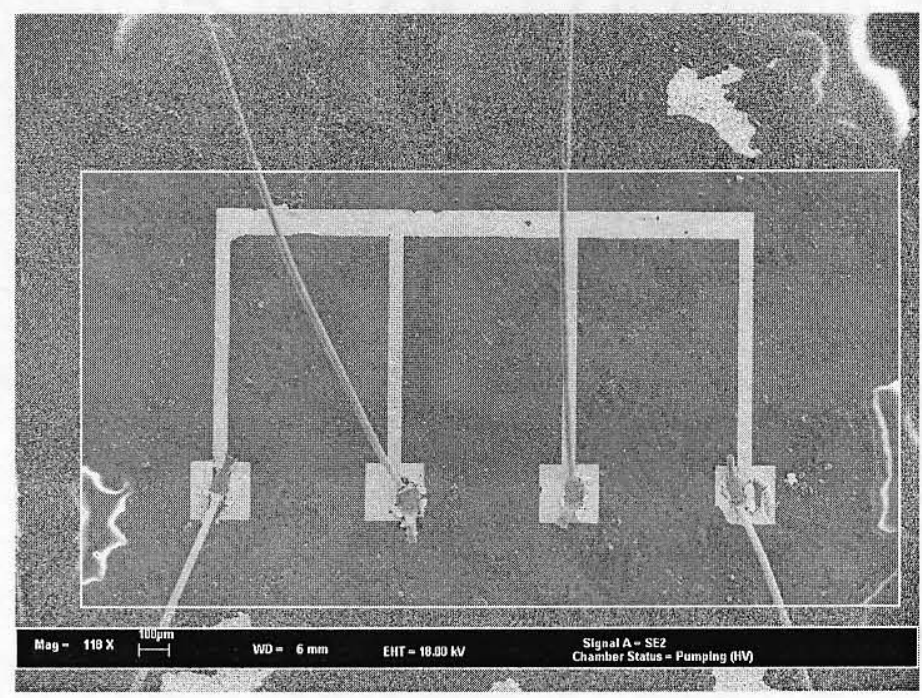

Fig.23: 4 pad test structure for $3 \omega$ method.

Fig.24 shows the schematic diagram of the measurement system of thermal conductivity. The control was realised using a PC with IEC-bus.

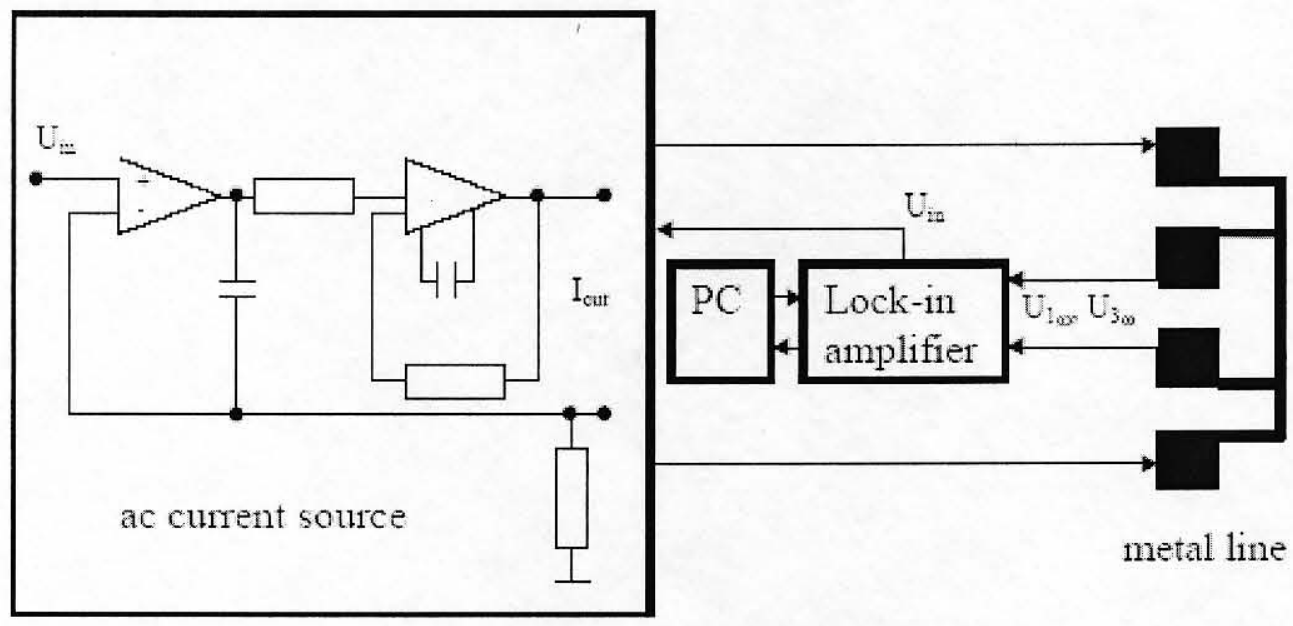

Fig. 24: Schematic diagram of the measurement system

Figure 24 shows the block diagram for the measurement. A digital lock-in amplifier such as a SR810 made (Fig. 21) by Stanford Research Inc. was selected. All the filters on the lock-in were turned off, and the dc coupled input mode was selected, to ensure the observation of a true frequency dependence of $V 3 \omega$. Before measuring the $3 \omega$ signal, the 
$R^{\prime} \circ$ or $180^{\circ}-$ if $R^{\prime} \times 0$ phase of the lock-in amplifier was adjusted to zero according to the $1 \omega$ voltage component. The phase angle of $V_{3 \omega}$ is then $-\varphi$ if according to Eq. (19). We used a simple electronic circuit (the lower panel of Fig. 21) to convert the $1 \omega$ sine wave voltage from the sine out of the lock in to an ac current, and then we fed the current into the specimen. Because the $3 \omega$ voltage signal is deeply buried in the $1 \omega$ voltage signal, a certain amount of dynamic reservation is required for the lock in if, in order to keep the simplicity of this method, one is not using a bridge circuit to cancel out the $1 \omega$ signal. We kept the dynamic reservation unchanged relative to the total magnification of the lock in during the entire measurement.

\section{Results and Discussion}

The experimental technique was tested with glass and Silicon substrate. The calibration was made by thermal estimation as given by Cahill [10]. This equation

$$
\Delta I=P_{1}: \pi /\left[-0.5 \ln (2 \omega)-0.5 \ln \left(\lambda, p c_{0} b^{2}\right)-\eta\right] . \eta=\text { const }
$$

[11] is valid for the substrate. In this simplest case, there is only the substrate. With the constant $\boldsymbol{\eta}$ the estimation can be fitted to the measurement.

With the equation

$$
\left.\lambda=\left[V_{0 \omega}{ }^{2} \ln \left(f_{2} f_{1}\right)\right] \alpha / 4 \pi 1 R\left(V_{3 \omega 1}-V_{3 \omega_{2}}\right)\right]
$$

[12] the thermal conductivity of the glass wafer $\lambda=(1.2 \pm 0.07) \mathrm{W} / \mathrm{mK}$ for room temperature is investigated. The accuracy of the measurement is good comparing the measured value with the one provided by the manufacturer (Corning 7740 Pyrex $\_\lambda$ pyrex $=1.13 \mathrm{~W} / \mathrm{mK} .($ Fig.25) 


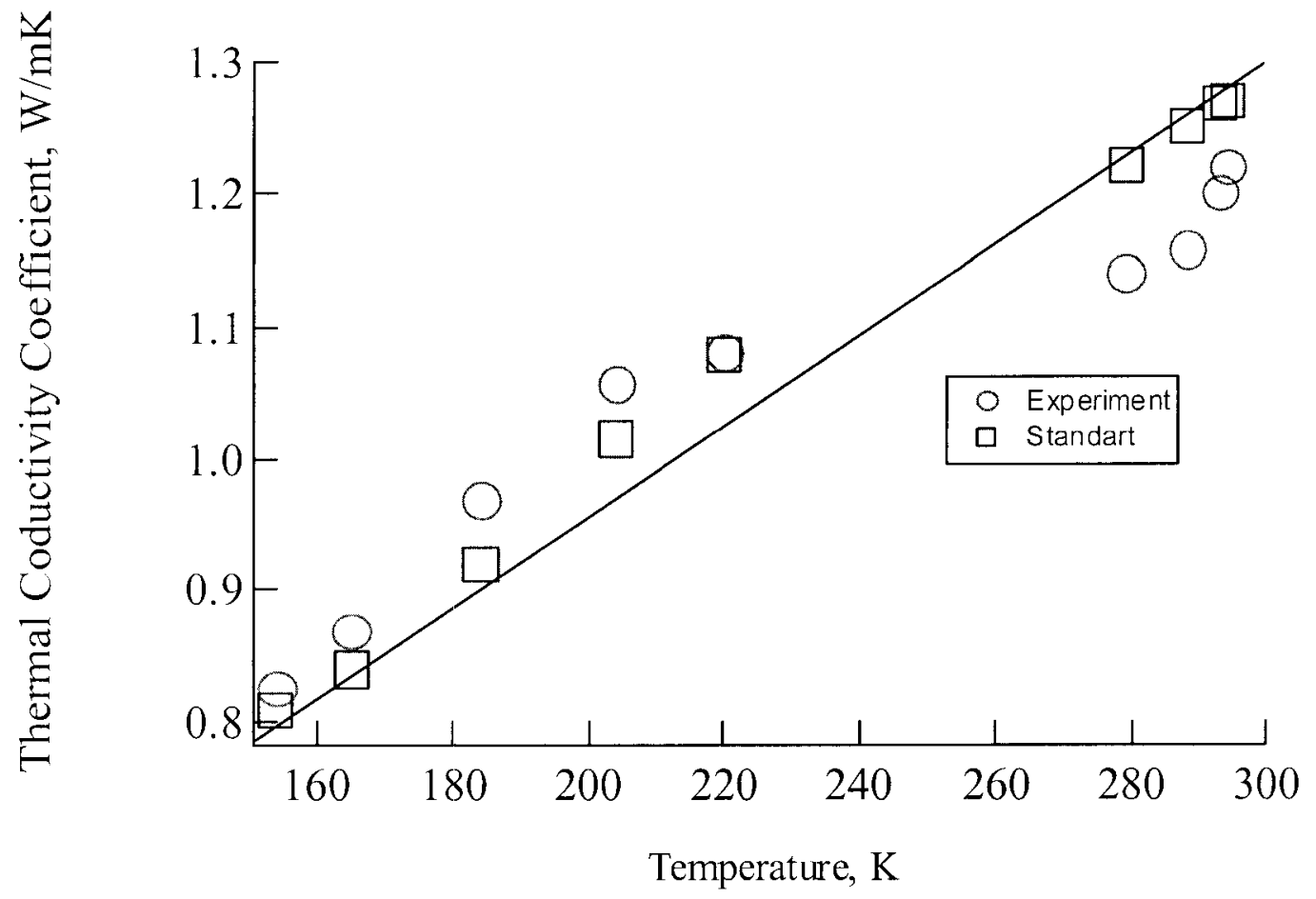

Fig. 25: Thermal conductivity coefficient $\lambda$ of quartz versus temperature $T$.

For Silicon sample and $\mathrm{SiO}_{2}$ the dielectric film is thin,. You can see the behavior on Fig 26

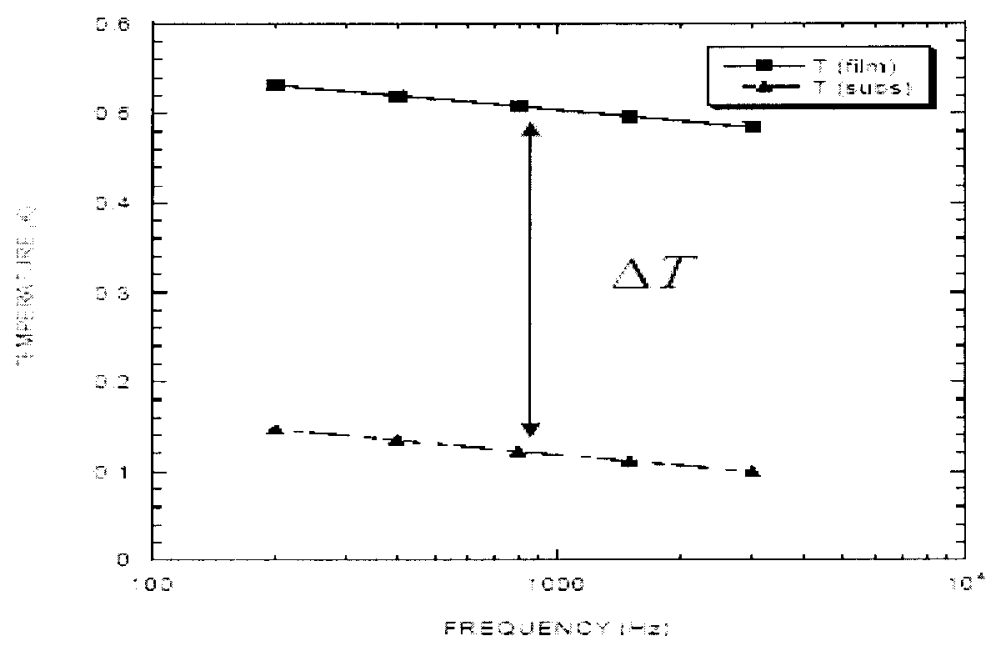

Fig. 26

- $\lambda_{s}: 145.6 \mathrm{~W} / \mathrm{mK} \lambda_{\text {filn }}: 1.0382 \pm 0.0015 \mathrm{~W} / \mathrm{mK}$ 
- Bulk (Literature value) Silicon [13]: $148 \mathrm{~W} / \mathrm{mK}$ Silicon Dioxide: $1.1 \mathrm{~W} / \mathrm{mK}$ $\mathrm{R}$ of the golf heater is changing with temperature changing (Fig. 27)

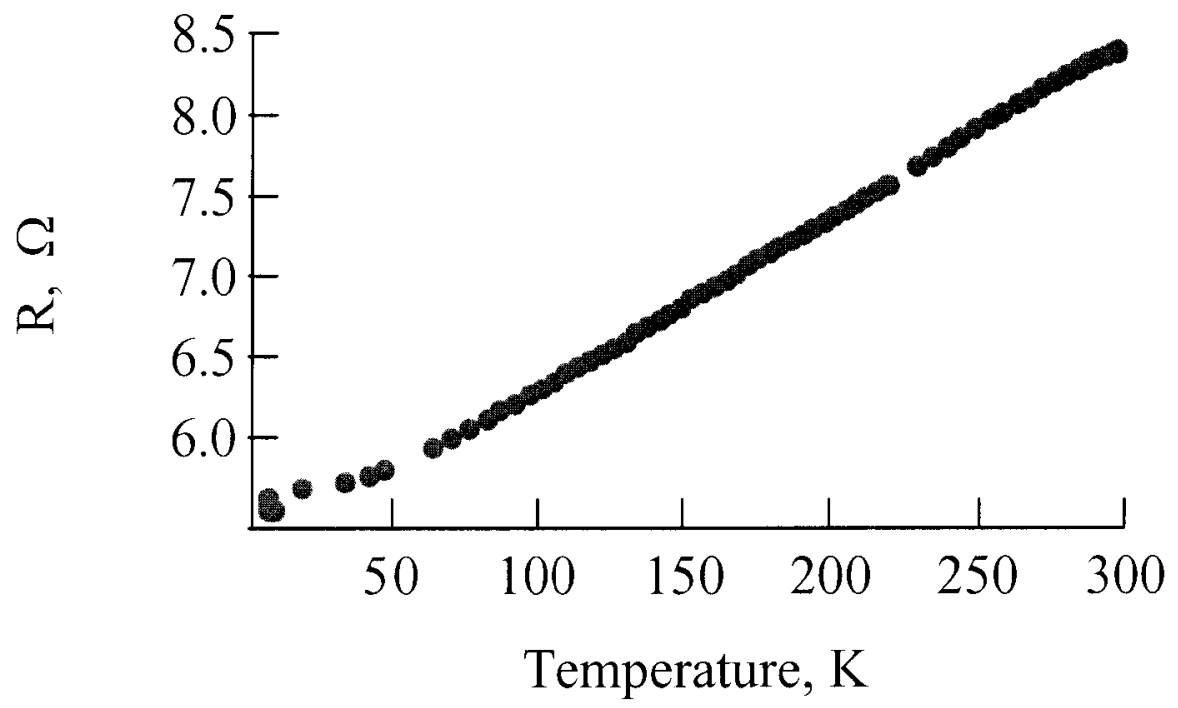

Fig. $27 \mathrm{Au} \mathrm{R}$ versus temperature

For a film-on-substrate sample Fig.28, the total temperature rise at the heater is composed of two parts: one is the temperature rise in the substrate and the other one is the temperature drop across the film. If the metal line is wide compared to the thickness of the film, the heat flow in the film will be mainly in the cross-plane direction, and the film simply adds a frequency independent thermal resistance: $(\mathrm{T}$ film $=\mathrm{Pld}$ film $/(2 \mathrm{~b} \lambda \mathrm{film})[11]$. This approach requires that the thermal conductivity of the film must be much smaller than that of the substrate. The temperature rise of quartz wafer $\left(\Delta T=4 V_{3 \omega} /\left(\alpha V_{l \omega}\right)\right.$ [12]) was calculated depending on the frequency (fig. 29). The estimation corresponds with the measurement if it is calculated with a thermal conductivity $\lambda$ quartz $=1.2 \mathrm{~W} / \mathrm{mK}$ between 5 and $2000 \mathrm{~Hz}$. The thermal conductivity of the $\lambda$ quartz $=(1.2 \pm 0.3) \mathrm{W} / \mathrm{mK}$ is in good agreement with the literature value of $1.2 \mathrm{~W} / \mathrm{mK}[14]$. 


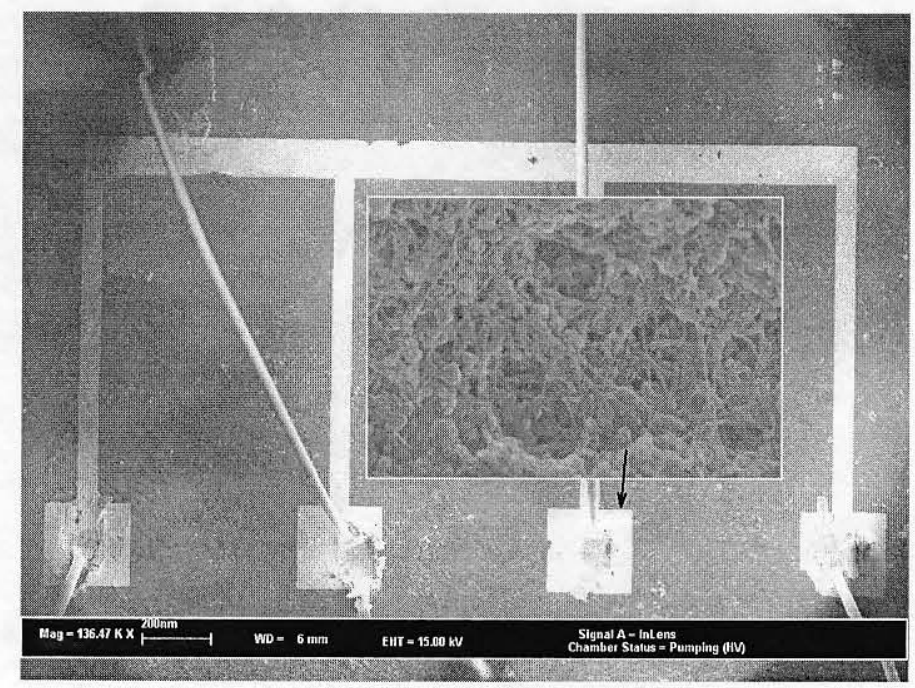

Fig.28: a carbon film-on-quartz substrate sample

We know that $\mathrm{V}_{3 \omega}=\mathrm{I}_{1} * \alpha / 2 * \Delta \mathrm{T}$. So, then we can calculate $\Delta \mathrm{T}$.

We can calculate $\Lambda$ for carbon thin film on quartz substrate from graph presented Fig. 30 .

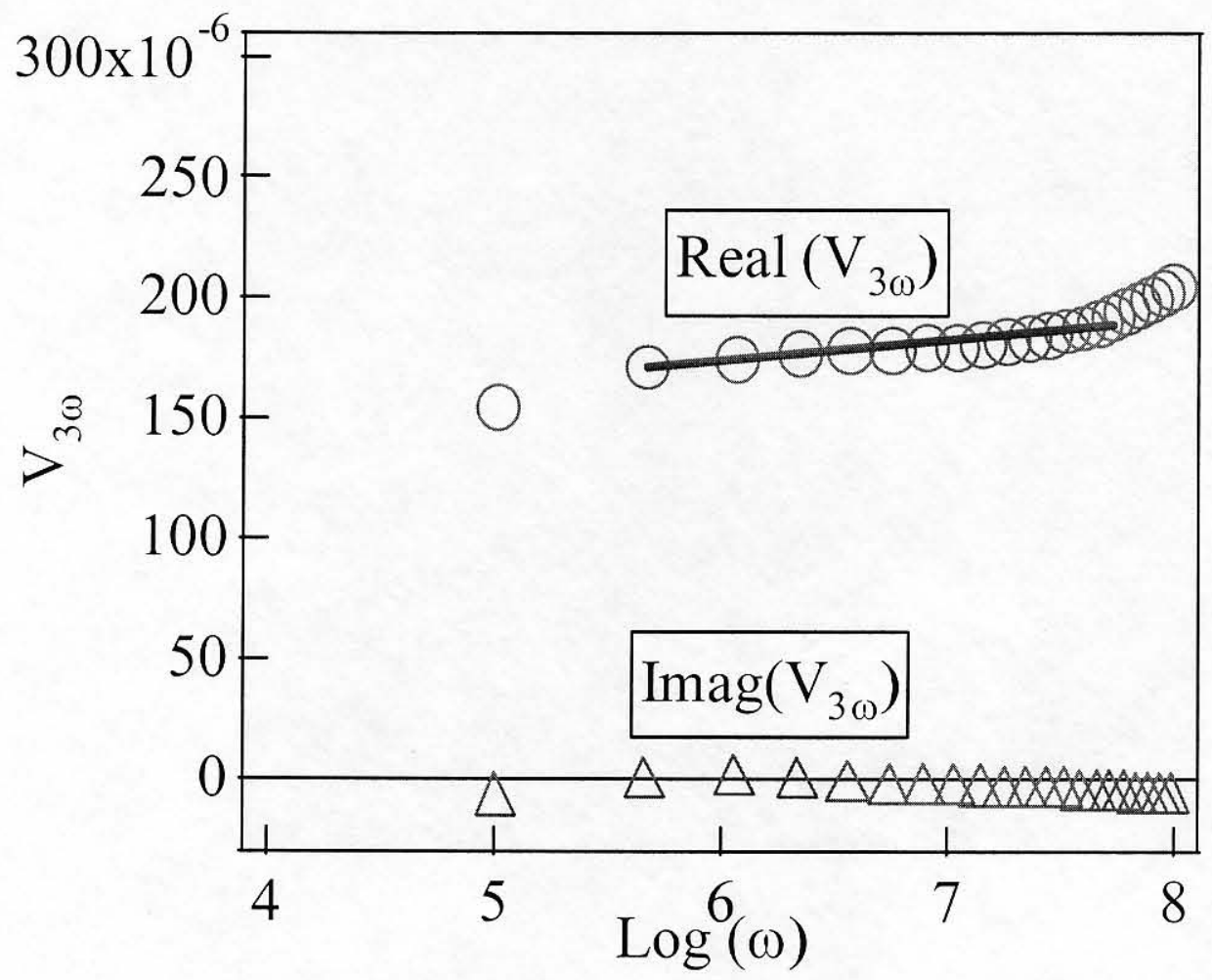

Fig. 29: dependence of $V 3 \omega$ from frequency. 


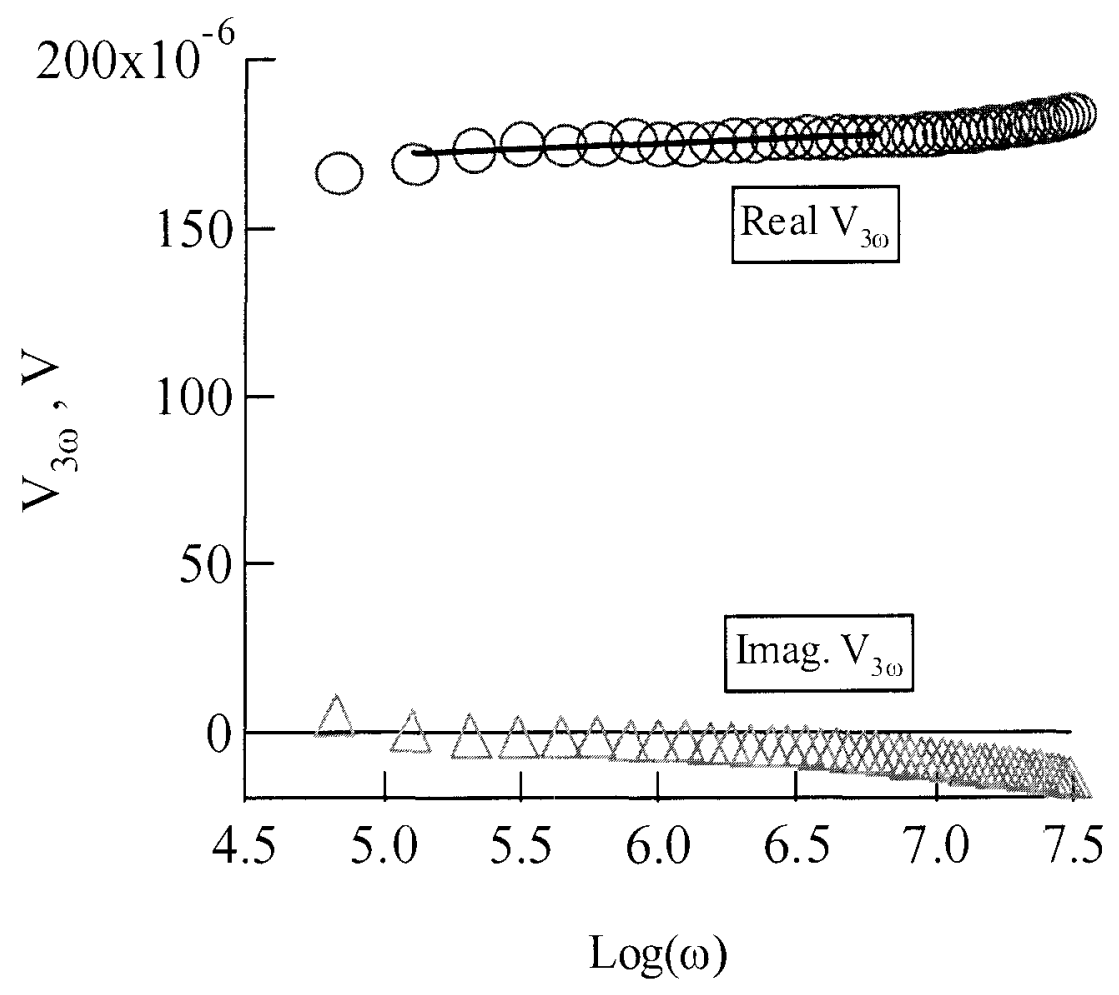

Fig. 30: $V_{3 \omega}$ versus $\log ($ freq) for Carbon thin film at room temperature.

And next graph (Fig.31) is showing the temperature dependence of $\lambda$ carbon thin film versus temperature $\mathrm{T}, \mathrm{K}$ 


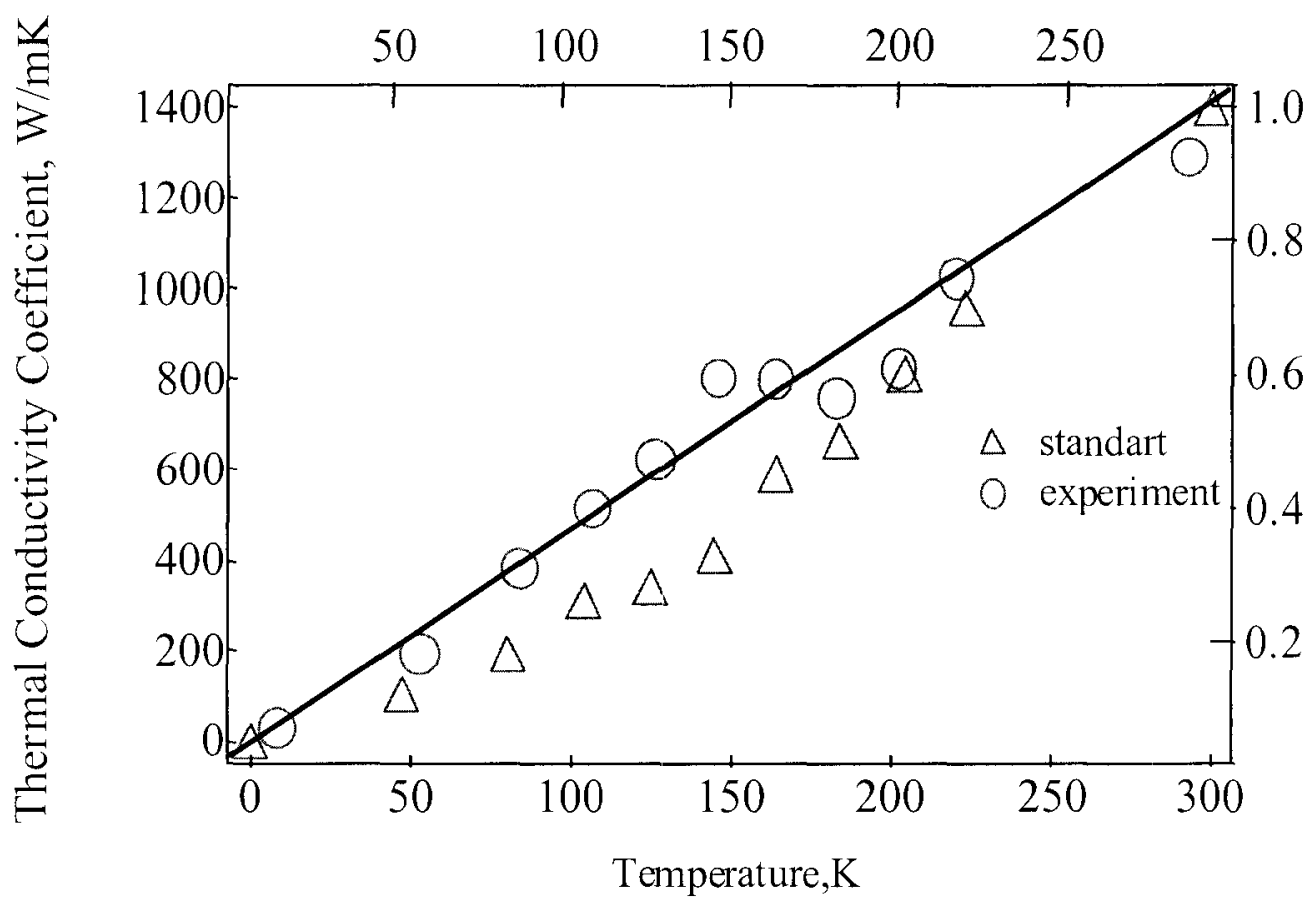

Fig. 31: $\lambda$ carbon thin film versus temperature.

The table below (Table 2) has experimental points for Carbon SWNT which represented on a Fig. 31

\begin{tabular}{|l|l|}
\hline$\underline{\boldsymbol{T} \boldsymbol{K}}$ & $\underline{\boldsymbol{\lambda}, \boldsymbol{W} / \boldsymbol{m} \boldsymbol{k}}$ \\
293 & 1293.9 \\
270 & 830.245 \\
220 & 1025.11 \\
202 & 826.319 \\
183 & 761.124 \\
164 & 801.136 \\
146 & 804.508 \\
127 & 626.32 \\
107 & 517.58 \\
84 & 386.54 \\
53 & 197.02 \\
8 & 32.04 \\
\hline
\end{tabular}

Table 2. $\lambda$ carbon thin film dependence of $\mathrm{T}$ for quartz sample

Now let compare our result by $3 \omega$ method with result for absolutely the same sample but by thermocouple method. Differential thermocouples were attached directly to the sample and the constantan rod to measure the temperature drops across each. A heater at one end 
of the constant rod provided a heat current through the rod and sample to the cold temperature stage; the thermal conductivity could then be calculated by comparing the temperature drops across the sample and the constantan rod. So, by this method $\lambda$ carbon thin film $=1750 \mathrm{~W} / \mathrm{mK} .-5800 \mathrm{~W} / \mathrm{mK}$

Figure 31 represents the measured thermal conductivity $\lambda$ of a representative SWNT sample as a function of temperature from $300 \mathrm{~K}$ to $8 \mathrm{~K}$. From $350 \mathrm{~K}$ to $40 \mathrm{~K}, \lambda$ decreases smoothly with decreasing temperature with very little curvature. The inset to Fig. 8 shows the low-temperature behavior. Near $30 \mathrm{~K}, \lambda(T)$ changes slope; below this temperature, $\lambda(T)$ is strictly linear in temperature and extrapolates to zero at $T_{0} . \lambda$ displayed identical temperature dependence in all mat samples, as well as the sintered sample. Thus we conclude that the measured thermal conductivity reflects the intrinsic thermal conductivity of nanotube bundles rather than sample-dependent effects such as the junctions between bundles.

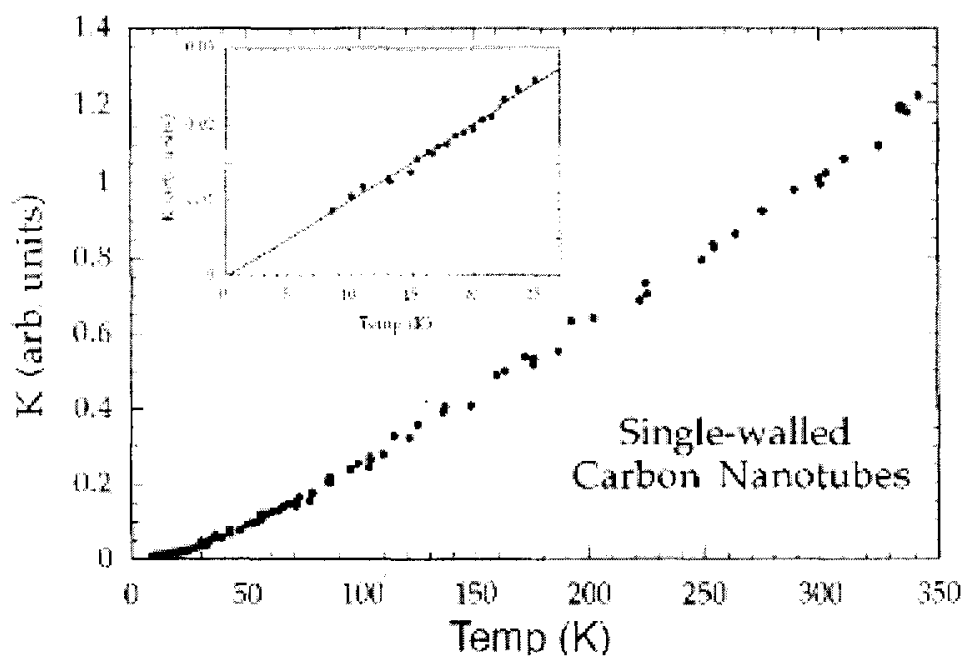

Fig.32: Temperature-dependent thermal conductivity of single-walled nanotubes. The thermal conductivity decreases smoothly from $350 \mathrm{~K}$ to $8 \mathrm{~K}$, and displays a change of slope near $30 \mathrm{~K}$. The inset shows the low-temperature behavior in greater detail. The solid line is a linear fit to the data below

$25 \mathrm{~K}$, and extrapolates to zero at zero temperature. 
The observed thermal conductivity displays a temperature dependence that is markedly different from that of graphite,[15,16] even though both materials are composed of graphitic sheets. In high-quality graphite, the $a b$-plane thermal conductivity, which is dominated by acoustic phonons, varies as $T[17-18]$ up to $\sim 150 \mathrm{~K}$, at which point phononphonon umklapp scattering causes $\mathrm{k}$ to decrease rapidly with increasing $T$. Highly pure graphite samples can have a thermal conductivity near $6000 \mathrm{~W} / \mathrm{m}-\mathrm{K}$ at the peak and 2000 $\mathrm{W} / \mathrm{m}-\mathrm{K}$ at room temperature.

All data we got by using software LabView 6.2 Fig.33
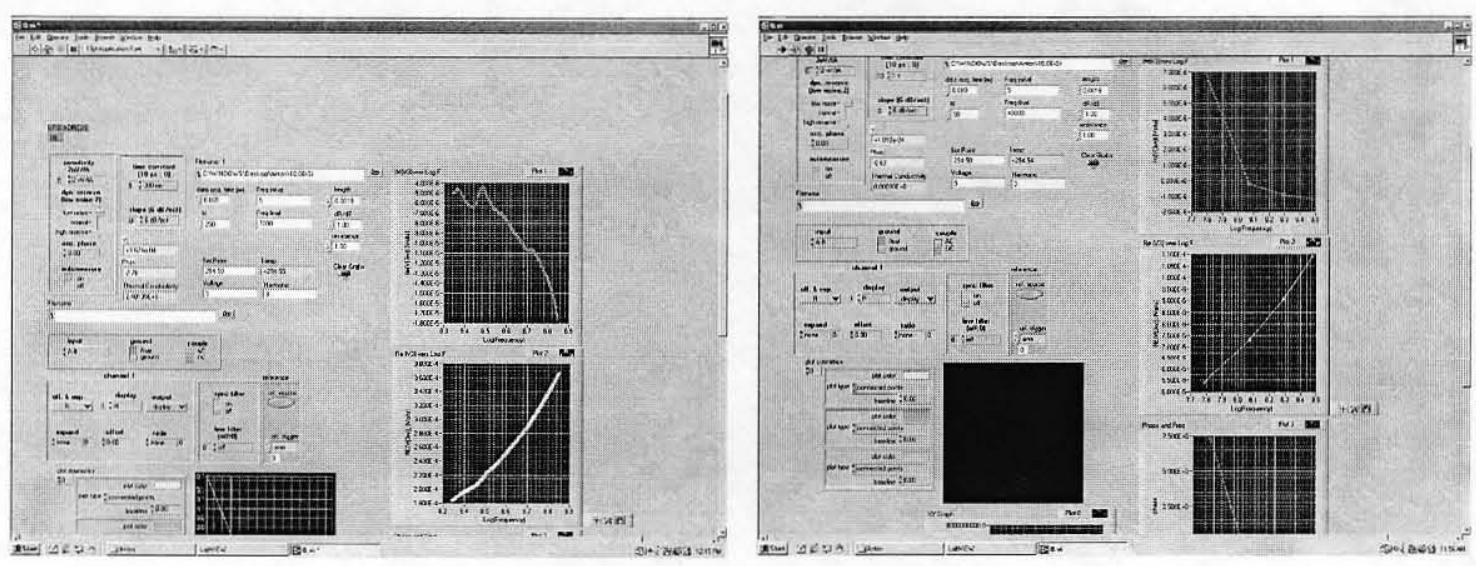

Fig. 33 


\section{CHAPTER IV}

\section{Conclusion}

In this thesis work we present results of temperature dependence of thermal conductivity $(\kappa(T))$ of thin films of SWNTs using a novel technique. This is the first time 3-0 technique has been used for $\kappa(\mathrm{T})$ measurements in SWNTs and shows excellent agreement with results obtained by steady state techniques. We explored a 3- $\omega$ method for measuring the thermal conductivity of silicon, silicon dioxide, quartz and SWNT thin films. We can measure $\mathrm{k}(\mathrm{T})$ of the specimen to an accuracy of $8 \%-15 \%$ with possibility of achieving a higher accuracy. A successful measurement relies on properly choosing the dimensions of the specimen so that one can have a large enough 3- $\omega$ voltage, yet maintain a convenient working frequency range and keep required conditions satisfied. Also, it was found out that Thermal Conductivity depends on thickness of material. These changes are probably connected by the change of fractal structure when compressing the sample. A few concrete samples were analyzed in detail. The values of thermal conductivity coefficients are in a good agreement with results obtained from different methods. The values of thermal conductivity were compared with existing data. 


\section{REFERENCES}

[1] Zhong Lin Wang, CHARACTERIZATION OF NANOPHASE MATERIALS.

[2] J. Chem. Phys., Vol. 119, No. 13, 1 October 2003.

[3] J. Phys. Chem. B 2003, 107, 4527-4531.

[4] P. Holister, J. W. Weener, C. Roman Vas, T. Harper; Cientifica.

[5] Appenzeller, J.; Martel, R.; Radosavljevic, M.; Heinze, S.; Avouris, P. Source: 2003

Third IEEE Conference on Nanotechnology. IEEE-NANO 2003. Proceedings (Cat.

No.03TH8700), 2003, pt. 2, p 236-9 vol.2.

[6] Jae-Yoo Kim, Moonhee Kim, Jong-Ho Choi. , Synthetic Metals 139 (2003) 565568.

[7] L.R.Holland, J. Appl.Phys. 34, 2350(1963); D. Gerlich, B. Abeles, and R.E Miller, ibial. 36,76(1965).

[8] D.G. Cahill, H.E. Fisher, T. Klitsnerr, E.T. Swartz, and R.O. Pohl, J.Vac. Sci.

Technol. A 7, 1259 (1989); D. G. Cahill, Rev. Sci. Instrum. 61, 802 (1990).

[9] L. Lu, W. Yi, and D. L. Zhang, VOL72, No7, JULY 2001.

[10] D.G. Gahill, K.E. Goodson, A. Majumbar, "Thermal Conductivity Mesurement from 30 to $750 \mathrm{~K}$ : the $3 \omega$ Method", Review of Scientific Instruments, vol. 61, no. 2, p.223-241, 2002

[11] S.M. Lee, D.G. Cahill, J.Appl. Phys. 81 (1997), 2590. 
[12] D.G. Cahill, Rev. Sci. Instrum. 61 (1990), 802.

[13] C. Hu, M. Morgen, P.S. Ho, A. Jain, W.N. Gill, J.L. Plawsky, P.C. Wayner, Appl. Phys. Lett. 77 (2000), 145.

[14] A. Jacquot et. al., J. Appl. Phys., 91 (7) (2002), 4733.

[15] G. W. C. Kaye and T. H. Laby, Tables of Physical and ChemicalConstants, 16th ed. (Longman, London, 1995).

[16] B. T. Kelly, Physics of Graphite. (Applied Science Publishers, London, 1981).

[17] S. Iijima, Nature (London) 354, 56 (1991).

[18] Rodney S. Ruoff and Donald C. Lorents, Carbon 33, 925 (1995). 


\section{CURRICULUM VITAE}

NAME: Anton Nickolaevich Sidorov

ADDRESS: Department of Physics

102 Natural Sciences Building

University of Louisville

Louisville, KY 40292.

DOB: $\quad$ Perm, Russia - August 19, 1981

EDUCATION

\& TRAINING: B.S, Physics

Perm State University, Russia

$1998-2003$

M.S, Physics

Perm State University, Russia

$1998-2003$

M.S, Physics

University of Louisville

Louisville, KY 40292

2003-2005 